\title{
On the optimality of diverse expert panels in persuasion games
}

\author{
Sourav Bhattacharya ${ }^{\mathrm{a}}$, Maria Goltsman ${ }^{\mathrm{b}}$, Arijit Mukherjee ${ }^{\mathrm{c}, *}$ \\ a Department of Economics, Royal Holloway, University of London, Egham, Surrey, TW20 OEX, UK \\ b Department of Economics, University of Western Ontario, 1151 Richmond St N, London, ON N6A 5C2, Canada

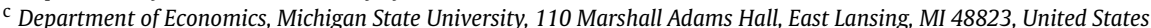

\section{A R T I C L E I N F O}

\section{Article history:}

Received 18 February 2016

Available online 22 November 2017

\section{JEL classification:}

D82

D83

\section{Keywords:}

Persuasion games

Panel design

\begin{abstract}
A B S T R A C T
We consider a persuasion game where the decision-maker relies on a panel of biased experts. An expert's preference is parameterized by his ideal action, or agenda. Common intuition suggests that more information is revealed if the panel includes experts with opposed agendas, because such experts will undo each other's attempts to conceal unfavorable information. In contrast, we show that recruiting experts with diverse agendas is optimal only if the correlation between the experts' types-i.e., whether they are informed or not-is above a threshold. Moreover, if the experts' types are independent, under mild assumptions it is optimal to recruit experts who have extreme but identical agendas. These findings suggest that the diversity of preferences must be considered in conjunction with the diversity of information sources, and it is generally sub-optimal to seek diversity in both dimensions.
\end{abstract}

(c) 2017 Elsevier Inc. All rights reserved.

\section{Introduction}

Decision-makers often rely on experts for information. However, the experts themselves may have preferences over the decision and strategically manipulate their advice. For example, suppose that the government is contemplating a policy for environmental regulation and seeks advice from a panel of experts on how stringent the regulation needs to be. An expert may be pro-industry and prefer to remove the regulation altogether, or pro-environment and prefer to have the most stringent regulation feasible. The ideological leanings of the potential members may be publicly known from their past records of public service and/or institutional affiliation. A natural question to ask in such an environment is the following: if the decision-maker could compose the panel by selecting experts with specific preferences, which experts should she choose? Should the panel consist of experts with conflicting ideological leanings, or of experts who share the same ideology?

\footnotetext{
We are very grateful to Gregory Pavlov for offering extensive comments on an earlier draft of this article. For their comments and discussions, we would also like to thank Heski Bar-Isaac, Andreas Blume, Brendan Daley, Rick Harbaugh, Thomas Jeitschko, Emir Kamenica, Wei Li, John Morgan, Debraj Ray, Michael Whinston and the seminar participants at International College of Economics and Finance, Michigan State University, National University of Singapore, New Economic School, Ohio State University, University of California-Riverside, University of Montreal, University of New South Wales, University of Pittsburgh, University of Toronto, Game Theory Society World Congress (Istanbul, 2012), Stony Brook Game Theory Conference (2013), 9th Annual Conference on Economic Growth and Development (New Delhi, 2013) and CIREQ Economic Theory Conference (2013). All errors that remain are our own.

* Corresponding author.

E-mail addresses: sourav.bhattacharya@rhul.ac.uk (S. Bhattacharya), mgoltsma@uwo.ca (M. Goltsman), arijit@msu.edu (A. Mukherjee).

URLs: http://personal.rhul.ac.uk/ucte/003/ (S. Bhattacharya), http://economics.uwo.ca/people/faculty/goltsman.html (M. Goltsman), http://www.amukherjee.net (A. Mukherjee).
} 
Common intuition suggests that a panel of experts with opposed preferences is more conducive to information revelation (see, e.g., Milgrom, 2008; Sward, 1989). The perceived benefit of such a panel is that the competing experts may undo each other's attempts to conceal unfavorable information. The literature on persuasion games has also primarily focused on the settings where the preferences of the informed parties are opposed in some sense (notable examples include Milgrom and Roberts, 1986; Lipman and Seppi, 1995; Shin, 1994, 1998; Glazer and Rubinstein, 2001; and Chen and Olszewski, 2014).

In this article, we argue that the above intuition favoring diversity may be misleading and the question of optimal panel design is considerably more nuanced. We compare a panel composed of experts with opposite preferences (which we call the diverse panel) with one where experts have identical preferences (the homogeneous panel), allowing for correlation in expert types, i.e., whether or not the experts possess the decision-relevant information. Our main result is that the diverse panel is optimal when such correlation is high and a homogeneous panel is optimal when the correlation is low. In particular, when expert types are independent, a homogeneous panel outperforms the diverse panel in a broad range of environments, including ones that are commonly assumed in the literature.

Notice that correlation in expert types may arise from the similarity in information sources accessed by the experts or similarity in methodology used in analyzing the available data. Thus, our result indicates that it is important to distinguish between two kinds of diversity among the experts-diversity in their preferences and diversity in their information sources-and it is typically sub-optimal to seek diversity in both dimensions.

In a related article, Bhattacharya and Mukherjee (2013; henceforth BM) develop a tractable framework to study persuasion with multiple experts. They also present an example where the decision-maker is better off under a homogeneous rather than a diverse panel. The current article attempts to uncover the key drivers behind the optimal panel design and explores conditions under which each type of panel-diverse or homogeneous-may be optimal.

In our model that closely follows BM's framework, a decision-maker has to choose an action in the unit interval [0,1]. The optimal decision from the decision-maker's point of view is denoted as the state of the world. Her objective is to minimize the loss which is an increasing function of the decision error, i.e., the distance between the state and the action. The decision-maker chooses her action based on verifiable reports from the two experts. The experts have state-independent and monotonic preferences over the decision-maker's action. In particular, an expert's preference is identified by his "agenda" or most preferred action (which can be 0 or 1 ). Each expert is privately informed of the state with a probability which we refer to as his "quality". Expert types (informed or uninformed) may be correlated. We assume that the information is hard in the sense that the state cannot be incorrectly reported. An uninformed expert must admit ignorance while an informed expert has the choice to either report the state or to pretend to be uninformed. The decision-maker takes the action that maximizes her expected payoff, given her posterior belief about the state based on the reports from the two experts.

In this framework, we ask the following question: If the decision-maker could choose the experts (at the beginning of the game) based on their agendas, what factors drive her choice and when is each type of panel-diverse (experts with respective agendas 0 and 1 ) and homogeneous (both experts with the same agenda)-likely to be optimal?

The equilibrium in the disclosure game is characterized by the decision-maker's "default action", i.e., the action chosen endogenously when neither expert reveals the state. Each informed expert chooses to reveal the state if doing so gives him a payoff higher than the default, but chooses to pool with the uninformed otherwise. Thus, an expert with agenda 0 reports only those states that are smaller than the default while the one with agenda 1 reports only the states that are larger than the default. This characterization gives rise to a tradeoff between the diverse and the homogeneous panels.

We illustrate this tradeoff by comparing the diverse panel with the left-homogeneous panel (where both experts have agenda 0). In the diverse panel, the experts jointly cover the state space, and if both experts are informed, each state is reported by exactly one of the two experts. Thus, each state is reported with a probability that is equal to the quality of an expert. On the other hand, in the left-homogeneous panel, each (informed) expert reports all states that are smaller than the default. Therefore, ex-ante, each state left of the default is reported with the probability that at least one of the two experts is informed, but the states higher than the default are never reported. Moreover, the default action in the left-homogeneous panel is higher than that in the diverse panel: If both experts in a left-homogeneous panel plead ignorance, the decision-maker rationally places more weight on the expert(s) finding the state to be adverse rather than both experts simultaneously failing to observe the state.

Hence, compared to a diverse panel, in a homogeneous panel the decision-maker learns the state with a high probability if the state lies in a larger subset of the state space, but the states outside this subset are never revealed. The homogeneous panel is therefore more likely to be effective if the corresponding default action is sufficiently extreme (i.e., far enough from the common agenda of the two experts) implying that the set of states over which revelation improves (compared to the diverse panel) is large enough.

The optimal panel is ascertained by comparing the observability and associated losses over different parts of the state space, which, in turn, depend on the decision-maker's risk attitudes, the distribution of the state and the experts' types, and the location of the default actions (an equilibrium object in itself) under the two types of panels. We present two key results that highlight the role of these factors in driving the optimal panel choice.

First, we show that (Proposition 2) there is a threshold of correlation between the experts' types above which the diverse panel is optimal and below which one of the two homogeneous panels is optimal. The result follows from the simple observation that with an increase in correlation, the value of having both experts report over the same set of states goes down while the value of both reporting over different sets of states remains unaffected. 
Recall that the correlation in expert types can be interpreted in terms of the degree of similarity in the experts' information sources. Thus, the above result indicates in the optimal panel design there is a tension between the diversity in agenda and diversity in information sources. However, it is worth noting that in reality, it is hard to disentangle these two sources of diversity. An expert's background and training may not only shape his methodology and information sources he tends to access, but are also likely to influence his world-view. ${ }^{1}$ Therefore, when a homogeneous panel is replaced by a diverse panel, we may have two kinds of change: a change from similar to opposite preference or ideology, and a change from common to diverse information sources. Our result shows that these two changes have opposite effects on the relative effectiveness of a diverse panel.

Next, we study how the correlation threshold derived in Proposition 2 varies with the model parameters. In particular, we seek conditions under which this threshold is strictly positive as it informs us on optimal panel design in a natural benchmark case: experts with independent types. We show that (Proposition 3) if the expert types are independent, a homogeneous panel is optimal irrespective of the quality of experts if the probability density of the state is log-concave and the decision-maker's loss function satisfies decreasing absolute risk aversion (DARA). ${ }^{2}$ Notice that these conditions are fairly standard in the sense that they are satisfied by a lot of environments commonly assumed in the literature, e.g., (truncated) normal or uniform density of the state and quadratic losses.

To see the intuition, let us compare the diverse panel with the left-homogeneous panel. Recall that the default action is higher in the latter panel. Now, the decision-maker gains from a higher default action as: (i) it expands the set of states reported by both experts and reduces the set of unreported states, and (ii) it reduces the decision error arising in the unreported states above the default. But a higher default action also increases potential decision errors that may occur if a very low state goes unreported in the event that both experts are uninformed.

The two conditions in Proposition 3 jointly strengthen the advantage and dampen the disadvantage of a more extreme default action in a homogeneous panel. Log-concavity of density precludes situations where the prior distribution is so concentrated around a particular state that irrespective of the panel configuration, the default must lie close to that state. Moreover, a log-concave density puts relatively less weight on extreme states. Such "thin-tailed" density coupled with moderate risk aversion ensures that the larger decision errors in a homogeneous panel remain relatively inexpensive.

While log-concavity of density and DARA loss function for the decision-maker are jointly sufficient for the optimality of a homogeneous panel (with independent experts), these conditions are not necessary. However, we provide examples to argue that if these conditions are not met, the diverse panel can turn out to be optimal. We further show that, under appropriate conditions, the homogeneous panel becomes less likely to be optimal as the density function becomes less dispersed or the decision-maker becomes more sensitive to large errors.

Finally, we explore the optimal panel design for an arbitrary level of positive correlation. ${ }^{3}$ We show that under quadratic loss and log-concave and symmetric density, a homogeneous panel is optimal for an intermediate range of quality and the diverse panel is optimal otherwise (Proposition 4). This result follows from the observation that in a homogeneous panel, the marginal value of the second expert with the same preference is rather modest if either the first expert is already very likely to be informed or the experts are of low quality to begin with.

Related literature: Some of the earliest contributions to the literature on persuasion games, such as Milgrom (1981), Grossman (1981), and Milgrom and Roberts (1986), argue that under verifiable messages, if the expert is known to possess the relevant information, in equilibrium, a skeptical decision-maker can extract all information. ${ }^{4}$ However, this result is sensitive to the assumptions about the expert's information: if there is a positive probability that the expert is uninformed, then an expert with adverse information can hide such information in equilibrium by selective disclosure (see, e.g., Dye, 1985; Jung and Kwon, 1988; Okuno-Fujiwara et al., 1990; Shavell, 1994; Shin, 1994). ${ }^{5}$ Our model makes extensive use of these ideas in the context of optimal design of expert panels.

The literature on optimal composition of expert panels in the persuasion setting is still nascent. Several authors have compared the efficacy of a diverse expert panel with a setting where only one expert (or the decision-maker herself) is responsible for gathering and revealing all information. Such studies typically conclude in support of using a diverse panel. For example, Shin (1998) compares a panel of two experts with opposing interests to one unbiased expert, and shows that the former may reveal more information. Dewatripont and Tirole (1999) make a similar comparison in a model with costly information acquisition and monetary transfers. They show that the use of opposing experts (or, "advocacy") allows for sharper incentives for information acquisition. More recently, Gentzkow and Kamenica (2017) present a general model

\footnotetext{
1 In the above example on environmental regulation, two industry lobbyists may use similar arguments and tap into a similar set of networks for drawing their information; similarly, two environmental scientists or activists would look at articles published in similar journals, and so on.

2 Since the decision-maker's payoff function is the negative of her loss function, the former exhibits increasing absolute risk aversion (IARA) when the latter exhibits DARA.

3 Trivially, under a negative correlation, the conditions in Proposition 3 continue to guarantee that a homogeneous panel is optimal irrespective of the experts' quality.

4 For an extension of this result to more general environments, see Seidmann and Winter (1997), Mathis (2008), Hagenbach et al. (2014). The literature on unverifiable messages with multiple senders also presents a similar theme. It argues that if experts are known to be informed of the state, then there is a fully revealing equilibrium for a wide range of parameters (Krishna and Morgan, 2001b; Battaglini, 2002).

5 Some other reasons that full disclosure may not take place in equilibrium are disclosure costs (Verrecchia, 1983), structure of provability (Dziuda, 2011), equivocal information (Perez-Richet, 2012), and non-monotonicity of the expert's preferences with respect to the decision-maker's actions (Giovannoni and Seidmann, 2007).
} 
of persuasion games and identify conditions on the information environment under which competition (weakly) increases information revelation. They show that given these conditions, if the experts' preference misalignment increases, it never reduces the amount of information revealed in equilibrium (under the Blackwell informativeness ordering).

A conclusion similar to ours is reached by Gerardi and Yariv (2008), who demonstrate that the optimal panel comprises experts with identical extreme preferences in order to provide the best incentives for costly information acquisition. ${ }^{6}$ A similar observation is made by Kartik et al. (2017): under certain conditions the experts' information acquisition decisions are strategic substitutes and the presence of other experts (like-minded or opposed) may reduce an expert's effort in information gathering. Consequently, the decision maker may prefer consulting just one (albeit biased) expert to using a panel of (possibly) opposed experts.

An intermediate position is occupied by Beniers and Swank (2004), who consider a model where the experts can acquire both verifiable and unverifiable information, and, unlike in our model, the experts have state-dependent preferences. They show that the committee should consist of similar experts, whose preferences are close to those of the decision-maker, if the cost of information acquisition is low, and of experts with opposing extreme preferences, if the cost is high.

Notice that all the above studies argue for similar panels in terms of sharper incentives for information acquisition. In contrast, we assume the availability of information to be exogenous, and show that the relative efficiency of similar panels may stem from stronger incentives for information revelation.

The rest of the article is organized as follows. Section 2 presents the basic model, and the equilibrium of the persuasion game is characterized in Section 3. The question of optimal panel design is explored in Section 4 . Section 5 discusses some extensions of our model, and the final section draws a conclusion. Appendix A contains proofs not provided in the main text, and Appendix B contains some more results.

\section{The model}

Our model closely follows the environment described in BM. Consider a decision-maker who must take an action $y \in$ $Y=[0,1]$ based on the underlying state $\theta \in \Theta=[0,1]$. The decision-maker prefers to choose an action that minimizes her loss $v(|y-\theta|)$; we will assume that $v(\cdot)$ is twice differentiable, $v(0)=v^{\prime}(0)=0, v^{\prime \prime}(0)>0$, and $v^{\prime}(z)>0, v^{\prime \prime}(z)>0$ for $z>0$. The decision-maker does not observe the state, which is distributed according to the distribution function $F(\theta)$. We assume that the associated probability density function $f(\theta)$ exists and is continuous.

The decision-maker can solicit information from a panel of two experts, $A$ and $B$. Each expert can be of one of two types: informed or uninformed. An informed expert observes the state perfectly, while the uninformed expert observes nothing. An expert's type is his private information. We assume that the probability of being informed, $\alpha$, is the same across the experts; i.e.,

$$
\alpha:=\operatorname{Pr}(\text { expert } A \text { is informed })=\operatorname{Pr}(\text { expert } B \text { is informed }),
$$

and we refer to $\alpha$ as the quality of an expert.

We further assume that the experts' types may be correlated. Formally, let $\tau_{i} \in\{0,1\}$ be the type of expert $i$, where $\tau_{i}=1$ if expert $i$ is informed and 0 otherwise. Further, denote $\operatorname{Pr}\left(\tau_{A}, \tau_{B}\right)=p_{\tau_{A} \tau_{B}}$. The distribution over the types can be defined in terms of the marginal $\alpha$ and a measure of correlation between the types $\rho:=p_{11} p_{00}-p_{10} p_{01}$. In particular, we have:

$$
p_{00}=(1-\alpha)^{2}+\rho, p_{11}=\alpha^{2}+\rho, \text { and } p_{10}=p_{01}=\alpha(1-\alpha)-\rho .
$$

Clearly, $p_{11}+p_{10}=p_{11}+p_{01}=\alpha$. To make sure that all probabilities are well-defined, we will assume that $\rho \in\left[\rho_{0}, \rho_{1}\right]$, where $\rho_{0}=-\min \left\{\alpha^{2},(1-\alpha)^{2}\right\}$ and $\rho_{1}=\alpha(1-\alpha)$. As the following examples illustrate, the correlation among the experts' types may stem from the inherent nature of the available information sources as well as from the nature of the experts' access to these information sources.

Example 1 (Positive correlation due to nature of information access). Suppose that there are $N$ sources, each of which independently reveals the state with probability $1-p$, for some $p \in(0,1)$. Suppose also that $N=2 n+s$, where expert $A$ has private access to the first $n$ sources, expert $B$ has private access to the next $n$ sources and both have access to the final $s$ sources. Then $p_{10}=p_{01}=p^{n+s}\left(1-p^{n}\right), p_{00}=p^{2 n+s}$, and therefore $p_{11}=1-p^{2 n+s}-2 p^{n+s}\left(1-p^{n}\right)$. So $\rho=p_{11} p_{00}-p_{10} p_{01}=p^{N}\left(1-p^{s}\right) ; \rho=0$ if $s=0$ (all sources are proprietary), and $\rho>0$ if $s \in\{1, \ldots, N\}$; note that $\rho$ is increasing in the number of common sources $s$, the total number of sources $N$ being fixed.

Example 2 (Positive correlation due to nature of information source). The experts are running their tests on the same data. The data can be amenable to testing ("good") with probability $p \in(0,1)$, and "bad" with probability of $1-p$. The data quality is unknown to the experts. The testing technology is the same for both experts. If the data is good, the probability that the

\footnotetext{
6 For other instances of this result (i.e., increased diversity between senders leads to a less informative outcome), see Banerjee and Somanathan (2001) and Newton (2014). However, the mechanisms driving this result in these articles are very different from ours.

7 Krishna and Morgan (2001a) and Cai (2009) also argue that increasing the committee size may reduce the decision-maker's payoff.
} 
test reveals the state is $q \in(0,1)$. If the data is bad, the test never reveals the state. Suppose that both experts are allowed to run their tests, and the outcomes of the tests are statistically independent conditional on data quality. Then $p_{11}=p q^{2}$, $p_{10}=p_{01}=p q(1-q)$, and $p_{00}=1-p+p(1-q)^{2}$, so $\rho=p q^{2}(1-p)>0$.

Example 3 (Negative correlation due to exclusive access to information source). Suppose again that there are $N$ sources of information, and each expert has exclusive access to $N / 2$ sources. Exactly one of the sources is fruitful in that it potentially reveals the state. The fruitful source reveals the state with probability $1-p, p \in(0,1)$. Each source is equally likely to be fruitful ex ante. Now we have $p_{00}=p, p_{10}=p_{01}=\frac{1}{2}(1-p)$, and $p_{11}=0$. So $\rho=-\frac{1}{4}(1-p)^{2}<0$.

Each expert $i(i \in\{A, B\})$ has a state-independent ideal action, or agenda, $x_{i} \in\{0,1\}$, and his utility $u_{i}$ depends on his loss $\left|y-x_{i}\right|$. Since we only use the fact that an expert's utility function is strictly decreasing in the loss, we assume $u_{i}\left(\left|y-x_{i}\right|\right)=-\left|y-x_{i}\right|$. Without loss of generality, let us assume that $x_{A} \leq x_{B}$. The agendas are commonly known. We will refer to an agenda profile $\left(x_{A}, x_{B}\right) \in\{0,1\} \times\{0,1\}$ as a panel. We will call the agenda profile $\left(x_{A}, x_{B}\right)=(0,1)$ the diverse panel, $\left(x_{A}, x_{B}\right)=(0,0)$ the left-homogeneous panel, and $\left(x_{A}, x_{B}\right)=(1,1)$ the right-homogeneous panel.

Before the decision-maker chooses her action, each expert sends a message $m_{i}(i \in\{A, B\})$ to the decision-maker. The messages are assumed to be verifiable. An uninformed expert must admit that he is uninformed, i.e., report $m_{i}=\emptyset$, but an informed expert has the option of either revealing the true state or feigning ignorance and pooling with the genuinely uninformed type; i.e., the message set for an informed expert in state $\theta$ is $\{\theta, \emptyset\}$. Given a profile of messages $m=\left\langle m_{A}, m_{B}\right\rangle$, the decision-maker takes an action $y(m) \in[0,1]$.

We use perfect Bayesian Equilibrium as the solution concept. ${ }^{8}$ Also, as $F$ is nonatomic, without loss of generality, we can restrict attention to pure strategies. The key focus of our analysis is to characterize the panel $\left(x_{A}, x_{B}\right)$ that minimizes the ex-ante loss of the decision-maker (given $\alpha$ and $\rho$ ). In other words, we seek to characterize the decision-maker's panel choice if she could form the panel by choosing two experts based on their publicly known agendas (keeping the probability distribution over expert types fixed).

A few remarks about the model are in order. First, notice the key difference between our model and that of BM: While BM allows for any compact and convex state space in $\mathbb{R}^{n}$, it assumes that the experts' types are independent. In contrast, we assume that the state space is a bounded interval in $\mathbb{R}$ but allow for correlated types. As we will discuss below, the correlation between types plays a salient role in our analysis, and the assumption of unidimensional state space enables us to clearly define the notion of opposing agendas between experts.

Second, though we limit attention to experts with "extreme" agendas (as $x_{i} \in\{0,1\}$ ), for the analysis of the optimal panel design this assumption does not entail any loss of generality. While a more general model could allow for moderate agendas (i.e., $\left.x_{i} \in[0,1]\right)$, BM shows that the decision-maker's ex-ante loss (in equilibrium) is always minimized when the agendas of both experts are in $\{0,1\} .^{9}$

Finally, we could allow for a more general reporting strategy for the experts à la Milgrom (1981): an informed expert may obfuscate his message by reporting a subset of the state space that contains the true state. Following Milgrom's unravelling argument, one can argue that whenever the experts have extreme agendas the set of equilibria under such general reporting strategies exactly coincides with the set of equilibria in our model. Hence, our analysis would remain unaltered even if we consider such a broader class of reporting strategies. ${ }^{10}$

\section{Equilibrium characterization}

In this section, we briefly state the key characteristics of the equilibrium of the persuasion game for a given panel $\left(x_{A}, x_{B}\right)$. The analysis below adapts the equilibrium characterization discussed in BM to our setting and highlights the trade-off associated with the optimal panel choice.

Observe that if at least one expert reveals the state (i.e., if $m_{i}=\theta$ for some $i \in\{A, B\}$ ), then the decision-maker optimally chooses $y(m)=\theta$. Thus, the decision-maker's strategy is completely characterized by her action $y^{*}:=y(\emptyset, \emptyset)$ when neither expert reports the state. We call $y^{*}$ the decision-maker's default action. On the other hand, the strategy of each informed expert $i$ is described by his revelation set $\Theta_{i}=\left\{\theta \in \Theta: m_{i}=\theta\right\}$, which is the set of states that he would report.

Also note that an informed expert $i$ 's choice of message matters only when the other expert does not report. Conditional on this event, expert $i$ induces the action $y=\theta$ by disclosing the state, and the default action $y=y^{*}$ by not disclosing. Thus, revealing the state is a best response for informed expert $i$ if and only if $\theta \in \Theta_{i}\left(y^{*}\right)=\left\{\theta \in \Theta:\left|y^{*}-x_{i}\right| \geq\left|\theta-x_{i}\right|\right\}$. ${ }^{11}$ In particular, if $x_{i}=0$, then $\Theta_{i}\left(y^{*}\right)=\left[0, y^{*}\right]$, and if $x_{i}=1$, then $\Theta_{i}\left(y^{*}\right)=\left[y^{*}, 1\right]$.

\footnotetext{
8 Note that our assumption of verifiable information on states implies that if an expert takes an off-equilibrium action that reveals $\theta$, the decision-maker's off-equilibrium belief must be degenerate at $\theta$.

9 While BM proves this result with independent expert types $(\rho=0)$, the proof extends straightforwardly to $\rho \in\left[\rho_{0}, \rho_{1}\right]$.

10 However, in this situation, panels with extreme experts may not be optimal. In particular, we cannot rule out the existence of partially informative equilibria with non-extreme expert panels that may lead to a higher payoff for the decision-maker than the extreme panels. Nevertheless, we are unaware of the existence of such a case.

11 Here we are focusing on equilibria where an expert always reveals the state whenever he is indifferent between revealing and concealing. As $F(\theta)$ is continuous, any alternative specification in this regard would not change the decision-maker's equilibrium payoff.
} 
Let us denote the equilibrium default action for the panel $\left(x_{A}, x_{B}\right)$ by $y_{x_{A} x_{B}}^{*}$. That is,

$$
\begin{aligned}
& y_{01}^{*}=\arg \min _{y \in[0,1]}(1-\alpha) \int_{0}^{y_{01}^{*}} v(|y-\theta|) d F(\theta)+(1-\alpha) \int_{y_{01}^{*}}^{1} v(|y-\theta|) d F(\theta), \\
& y_{00}^{*}=\arg \min _{y \in[0,1]}\left((1-\alpha)^{2}+\rho\right) \int_{0}^{y_{00}^{*}} v(|y-\theta|) d F(\theta)+\int_{y_{00}^{*}}^{1} v(|y-\theta|) d F(\theta), \\
& y_{11}^{*}=\arg \min _{y \in[0,1]} \int_{0}^{y_{11}^{*}} v(|y-\theta|) d F(\theta)+\left((1-\alpha)^{2}+\rho\right) \int_{y_{11}^{*}}^{1} v(|y-\theta|) d F(\theta) .
\end{aligned}
$$

The associated first-order conditions are given as:

$$
\frac{\int_{0}^{y_{x_{A} x_{B}}^{*}} v^{\prime}\left(y_{x_{A} x_{B}}^{*}-\theta\right) d F(\theta)}{\int_{y_{x_{A} x_{B}}^{*}}^{1} v^{\prime}\left(\theta-y_{X_{A} x_{B}}^{*}\right) d F(\theta)}= \begin{cases}1 & \text { if }\left(x_{A}, x_{B}\right)=(0,1) \\ 1 /\left((1-\alpha)^{2}+\rho\right) & \text { if }\left(x_{A}, x_{B}\right)=(0,0) \\ (1-\alpha)^{2}+\rho & \text { if }\left(x_{A}, x_{B}\right)=(1,1)\end{cases}
$$

The strict concavity of $v$ guarantees that the default action is unique under all panels.

Note that the default action in a diverse panel, $y_{01}^{*}$, is independent of $\alpha$ and $\rho$, and it is identical to the action the decision-maker would take if none of the experts were present. Two other salient characteristics of the equilibrium default action are given in the proposition below. (This result is based on propositions 2 and 4 in BM. We omit the formal proof for sake of brevity as the subsequent discussion illustrates the argument). As we will see later, these observations play a critical role in our subsequent analysis of the optimal panel design.

Proposition 1. (a) For any $\alpha \in(0,1)$ and $\rho \in\left[\rho_{0}, \rho_{1}\right], 0<y_{11}^{*}<y_{01}^{*}<y_{00}^{*}<1$. (b) For all three types of panels, the equilibria are outcome equivalent to the equilibria that would emerge if the decision-maker could commit to a default action before receiving the messages. ${ }^{12}$

The first part of the proposition states that the equilibrium default actions under the diverse and the two homogeneous panels can be unambiguously ranked. Intuitively, it reflects the rational skepticism of the decision-maker (Milgrom, 1981) as she updates her belief on the underlying state when the panel fails to reveal any information. First, consider the intuition for $y_{11}^{*}<y_{00}^{*}$ : Observe that in a right-homogeneous panel (similarly, left-homogeneous panel), both informed experts reveal the state if and only if is closer to 1 (similarly, closer to 0 ) than the default action. Thus, while updating her belief, the decision-maker puts more weight on the low states in the absence of reports from a $(1,1)$ panel than in the absence of reports from a $(0,0)$ panel. Conversely, the decision-maker puts more weight on the high states in the absence of reports from a $(0,0)$ panel than in absence of reports from a $(1,1)$ panel. Moreover, note that $y_{01}^{*} \in\left(y_{00}^{*}, y_{11}^{*}\right)$ as the absence of reports from a diverse panel can occur if either both experts are uninformed or if only one expert is informed and the state is further away from the agenda of the informed expert than the default action. Hence, the decision-maker puts less weight on states that are too high (similarly, too low) relative to the case of the $(0,0)$ panel (similarly, $(1,1)$ panel).

This result also allows us to see the trade-off between the diverse and the homogeneous panels. In the diverse panel, the two experts jointly "cover" the entire state space: the states below the default action are revealed by the expert with agenda 0 , and the states above by the expert with agenda 1 . In a homogeneous panel, on the other hand, the experts "cover" the same subset of the state space: for example, in the left-homogeneous panel, both experts will reveal the state if it is below the default action $y_{00}^{*}$, but none will if it is above. Also, the default action would be further away from agenda 0 than its diverse panel counterpart (i.e., $y_{01}^{*}<y_{00}^{*}$ ). The comparison between the diverse and the right-homogeneous panel is similar.

Hence, compared to the diverse panel, in a homogeneous panel the decision-maker learns the state with a greater probability if the state lies in a larger subset of the state space, but the states outside this subset are never revealed. In other words, consulting a diverse panel is similar to consulting a single expert of quality $\alpha$ who is "unbiased": if informed, he always reveals the state. In contrast, a homogeneous panel can be conceived as a single expert with a relatively higher quality, $1-\left((1-\alpha)^{2}+\rho\right)$, but one who is "biased": if informed, he reveals the state if and only if his agenda is closer to the state than to the default action. ${ }^{13}$

\footnotetext{
12 Here we assume that the decision-maker commits only to a default action, rather than to a mechanism that maps the reports to a default action.

13 We thank two anonymous referees for suggesting the aforementioned discussion on the intuition for Proposition 1 as well as the interpretation of the trade-off in terms of expert's quality and bias.
} 
(a) Diverse panel:

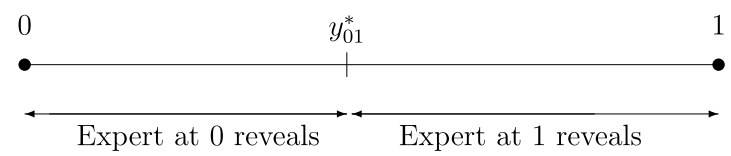

(b) Homogeneous panel (both experts have agenda 0):

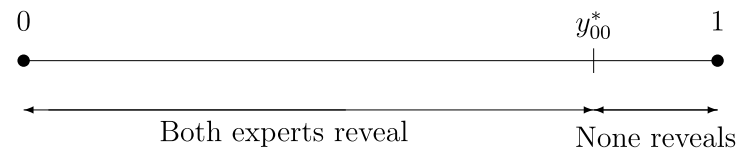

Fig. 1. The trade-off between the diverse panel (one expert at each end) and a homogeneous panel (both experts at 0).

This observation suggests that the optimal panel configuration will depend on the decision-maker's willingness to trade off the observability of different parts of the state space and the associated loss due to lack of report (see Fig. 1). But such loss, in turn, depends on her risk attitudes, the distribution of the state, and the location of the default actions (an equilibrium object in itself) under the two types of panels. Our analysis below attempts to clarify the role of these factors in driving the optimal panel choice.

Part (b) of the above proposition implies that we can we can treat the equilibrium default action as one that the decisionmaker would choose to minimize her ex-ante loss taking into account its impact on the experts' revelation strategy. This result directly follows from the observation that the first-order conditions for the optimal default action under commitment are identical to those in condition (1). As we will see below, it vastly simplifies our subsequent analysis of optimal panel design. ${ }^{14}$

\section{Optimal panel design}

Our first result establishes that a diverse panel is optimal if and only if the correlation between the expert types is above a threshold.

Proposition 2. For any $\alpha \in(0,1)$, there exists $\rho^{*} \in\left(\rho_{0}, \rho_{1}\right)$ (depending on the loss function $v$ and the density of the state $f$ ) such that a homogeneous panel is optimal if $\rho<\rho^{*}$ and a diverse panel is optimal otherwise.

To see the intuition behind this result, let us examine how the decision-maker's expected loss from consulting a lefthomogeneous panel and that from consulting a diverse panel change with $\rho$ (keeping $\alpha$ fixed). As discussed earlier, a diverse panel can be conceived as a single "unbiased" expert of quality $\alpha$, whereas a left-homogeneous panel is similar to consulting a single "biased" expert with a relatively higher quality, $1-\left((1-\alpha)^{2}+\rho\right)$, who reveals the state if and only if it is below $y_{00}^{*}$. When $\rho=\rho_{1}$, the quality difference between these two experts disappears. In a homogeneous panel the second expert becomes redundant since it cannot be the case that one expert is informed when the other is not. Thus, the choice between the two panels boils down to choosing between two experts of same quality $(\alpha)$, but in the case of homogeneous panel the expert is biased whereas in case of diverse panel he is not. Clearly, diverse panel becomes optimal.

As $\rho$ decreases, the expected loss from consulting a diverse panel stays the same-as the two experts' revelation sets are disjoint, the correlation in their types does not affect the decision-maker's payoff. The loss from a left-homogeneous panel, however, decreases due to two effects. First, there is a direct effect: as $\rho$ decreases, it increases the probability of one expert knowing the state conditional on the other one not knowing; this effect makes the second expert with agenda 0 more valuable. Second, there is an indirect effect: the first-order conditions (1) imply that as $\rho$ decreases, $y_{00}^{*}$ moves to the right, making the two experts in a left-homogeneous panel cover a larger part of the state space. A similar intuition applies in the comparison of the diverse and the right-homogeneous panel. Hence, as $\rho$ falls below a threshold, a homogeneous panel becomes optimal.

Proposition 2 suggests that when the experts' types are sufficiently likely to be the same, then it is optimal to have experts with diverse agendas. This may be the case when, for example, the experts mostly rely on the same sources. Note that this case corresponds to the environments illustrated in Example 1 where the number of common sources $s$ is sufficiently large as well as in Example 2 where the experts are running their independent investigations on the same data. If, on the other hand, the experts are likely to rely on different information sources, then a homogeneous panel is more likely to be the optimal design. This may be the case when, for example, the experts are using different methods to find

\footnotetext{
14 See Hart et al. (2017) and Sher (2011) for a more general treatment of equivalence between commitment and equilibrium outcomes in persuasion games with a single expert. Ben-Porath, et al. (2017) extend these results to multi-expert settings.
} 
out the state, only one of which can be fruitful (this case closely corresponds to the environment illustrated in Example 3). Thus, Proposition 2 highlights a tension between the two types of experts' diversity-diversity in agendas and diversity in information sources-and implies that seeking diversity in both dimensions may be undesirable.

Notice that in our analysis the correlation between experts' types is assumed to be exogenous and invariant to the design of the panel. While it is conceivable that the correlation in expert types can also change when we change the panel composition (e.g., like-minded experts may use the same information sources whereas opposing experts may use different ones), the exogeneity assumption helps us to tease out the role of correlation between expert types on the effectiveness of diverse and homogeneous panels. Moreover, in many real-life settings the decision-maker faces a choice of panel composition in terms of preferences, while the correlation in private information may be determined by the institutional norms that are not predicated upon the design of the panel.

For example, in many parliamentary and judicial settings, transparency laws or norms force the members of a panel to share all their documents and information sources. Such sharing of documents then causes a high correlation in expert information, with the level of correlation being determined by the nature of the question at hand and the availability of documents. This holds true irrespective of whether we consider the shared documents as discovered final information or simply as data on which each panel member runs his own inference. Importantly, such laws often exist prior to the selection of the panel. When any committee is selected in presence of such laws or norms, we can think of the problem of the decision-maker as deciding on the preference composition of the panel with the correlation in types being already given.

We can also make the following observation on how the correlation in types affects the decision-maker's equilibrium payoff. Fix $\alpha$, and suppose that the decision-maker can choose both $\rho$ and $\left(x_{A}, x_{B}\right)$. What value of $\rho$ maximizes the decisionmaker's payoff? It is interesting to note that the decision-maker's payoff is maximized when the types are as negatively correlated as possible (i.e., $\rho=\rho_{0}$ ) and one of the homogeneous panels is chosen. ${ }^{15}$ The argument is simple and a direct implication of the following two observations: First, from Proposition 2 we know that a homogeneous panel must be optimal for all $\rho \in\left(\rho_{0}, \rho^{*}\right)$, and second, the loss from any homogeneous panel monotonically increases in $\rho$, while that of the diverse panel is independent of $\rho$.

Next, we explore how $\rho^{*}$ depends on the loss function $v$ and the density of the state $f$. In particular, we seek conditions on $v$ and $f$ under which $\rho^{*}>0$, as the conditions would inform us on the optimal panel configuration when the expert types are statistically independent (i.e., $\rho=0$ ). Notice that the conditions on $v$ and $f$ for $\rho^{*}$ to be positive (negative) are exactly the conditions for the optimality of a homogeneous (diverse) panel when $\rho=0$. This analysis is important on its own merit. In many settings, independent expert types is a plausible assumption; moreover, it also serves as a benchmark as most of the extant literature on persuasion games maintains this assumption (see, e.g., Dewatripont and Tirole, 1999; Shin, 1998).

Unfortunately, for generic $v$ and $f$ functions, conditions that are both necessary and sufficient for the optimality of a given type of panel appear elusive. We first develop a set of sufficient conditions (Proposition 3) for the optimality of a homogeneous panel and then show that if these conditions are relaxed, the diverse panel could be optimal.

Proposition 3. If (i) $f(\theta)$ is log-concave and strictly positive on its domain and (ii) $v^{\prime \prime}(z) / v^{\prime}(z)$ is weakly decreasing, then $\rho^{*}>0$ for any $\alpha \in(0,1)$.

The main implication of the above result is that under the above conditions, a homogeneous panel is optimal irrespective of expert quality whenever the expert types are statistically independent (i.e., when $\rho=0$ ). The intuition behind this result can again be traced from the trade-off between quality and bias that we have mentioned earlier. Recall that a homogeneous panel can be conceived as single expert who is of relatively high quality (i.e., observes the state with probability 1 $\left.(1-\alpha)^{2}\right)$ but "biased". For example, under a left-homogeneous panel, the expert reveals the state only if it is below $y_{00}^{*}$. But the closer is $y_{00}^{*}$ to 1 , the smaller is the bias. Also notice that a homogeneous panel exposes the decision-maker to costlier errors-e.g., when the state is close to 0 and both experts are uninformed, a left-homogeneous panel leads to a larger error than a diverse panel as $y_{00}^{*}>y_{01}^{*}$. Also, the states closer to 1 are never revealed and necessarily lead to a loss. Thus, a homogeneous panel is more appealing to the decision-maker when (i) the default action is more extreme (i.e., $y_{00}^{*}$ is close to 1 and/or $y_{11}^{*}$ is close to 0 ) leading to a smaller bias and (ii) the errors in decision-making, $|y-\theta|$, particularly when the state is closer to the extreme, are not too costly.

The conditions invoked in the above proposition guarantee these two features of the decision-making process. The logconcavity of $f$ implies that the distribution is not too concentrated around any particular state and ensures that the default action under homogeneous panel is sufficiently extreme. Log-concavity also ensures that the density is "thin-tailed" in the sense that it does not put too much weight on the extreme states. This condition, along with the decreasing $v^{\prime \prime} / v^{\prime}$ condition, mutes the loss associated with the decision-making errors. Note that a weakly decreasing $v^{\prime \prime} / v^{\prime}$ implies that the decision-maker's loss function exhibits weakly decreasing absolute risk aversion (DARA); i.e., the loss function $v$ may not increase too rapidly with the magnitude of the decision error. The thin-tailed density and moderate risk tolerance of the

15 In a common value voting context, Ladha (1992) also concludes that negative or low correlation among expert's assessments improves decision-making. 
decision-maker ensure that errors in decision-making when the state is towards the extreme are not too costly for the decision-maker. Consequently, a homogeneous panel dominates its diverse counterpart. ${ }^{16}$

We conclude this section with two remarks that further clarify the implications and intuition of the above proposition. First, notice that the two conditions specified in Proposition 3 are quite familiar in the extant literature. The DARA loss function is commonly assumed (e.g., quadratic loss function) and so is log-concavity (e.g., normal (including truncated normal) and uniform distribution have this property). Thus, our finding suggests that when experts' types are independent (i.e., $\rho=0$ ), having experts with diverse agendas is suboptimal in a wide range of settings. This may be the case if, for example, the experts rely on diverse proprietary sources. (Note that in terms of the environment given in Example 1, this is the case when there is no common source, i.e., $s=0$.)

Second, notice that the two conditions in Proposition 3 are not individually sufficient; they must hold jointly to ensure the optimality of a homogeneous panel under independent expert types-if any one of these two conditions is relaxed, one can find parameters when the diverse panel becomes optimal even if $\rho=0$. The following two examples illustrate this point.

Example 4. Suppose that $v(|y-\theta|)=\exp \left(10(y-\theta)^{2}\right)-1, f$ is uniform, $\rho=0$ and $\alpha=1 / 2$. Note that $v^{\prime \prime} / v^{\prime}$ is not monotone; it decreases if $|y-\theta|<0.224$ and increases otherwise. Under the homogeneous panel $(0,0)$, the equilibrium default action is $y_{00}^{*}=0.5634$, and the decision-maker's expected loss is 1.07 (by symmetry, the loss in the same for the panel $(1,1)$ ). But under the diverse panel the default action is $y_{01}^{*}=0.5$, and the decision-maker's expected loss reduces to 1.06 .

Example 5. Let $v(|y-\theta|)=(y-\theta)^{2}$ and $\theta$ is drawn from $U[0,1]$ with probability $\frac{1}{4}$ and from $U\left[\frac{1}{2}-\delta, \frac{1}{2}+\delta\right]$ with probability $\frac{3}{4}$, where $0<\delta<\frac{1}{2}$. That is, pdf $f(\theta)$ is given as:

$$
f(\theta)= \begin{cases}\frac{1}{4}+\frac{3}{8 \delta} & \text { if } \theta \in\left[\frac{1}{2}-\delta, \frac{1}{2}+\delta\right] \\ \frac{1}{4} & \text { otherwise. }\end{cases}
$$

Also, suppose that $\rho=0$ and $\alpha=1 / 2$. In this case, diverse panel is optimal if $\delta<0.135$. In other words, the diverse panel is optimal if the distribution is concentrated around $1 / 2$, and the homogeneous panel is optimal otherwise. ${ }^{17}$

Example 4 considers a log-concave $f$ but relaxes the DARA condition on $v$. Notice that the large losses associated with large errors in decision-making tip the balance in favor of the diverse panel. In contrast, Example 5 maintains decreasing $v^{\prime \prime} / v^{\prime}$ but relaxes the log-concavity condition on $f$. Here the distribution is highly concentrated around the state which corresponds to the optimal default action $y_{01}^{*}$ for the diverse panel. Hence, a switch to a homogeneous panel may make the decision-maker worse off because the default action does not move much from its diverse panel counterpart and leads to large bias in reporting (e.g., in a $(0,0)$ panel the state is never revealed if it is larger than $y_{00}^{*} \approx 0.56$ ). Such a situation can be precluded by having the distribution sufficiently "spread out" on its support, which is ensured by the log-concavity of $f$.

This example also indicates that log-concavity is not necessary for the optimality of homogeneous panel: such a panel is indeed optimal for all $\delta>0.135$, even though the density is not log-concave for any $\delta \in(0,1 / 2)$. As $\delta$ increases, the concentration of probability density around $\theta=1 / 2$ decreases and, given the increased variance of the distribution, the decision-maker's loss increases under all types of panels. However, the homogeneous panel may lead to a smaller loss. As the concentration around $1 / 2$ decreases, the default action under a homogeneous panel moves further to the extreme, lowering the loss associated with such a panel. ${ }^{18}$

\section{Discussion}

In this section we present a few additional results that further illustrate how the primitives of the model may influence the optimal panel design. We also discuss the robustness of our findings to a few natural extensions of the model.

\subsection{Further remarks on the optimal panel design}

The intuition for Proposition 3 as discussed earlier naturally leads to the conjecture that the diverse panel is optimal for a wider range of $\rho$ (i.e., $\rho^{*}$ is lower) when large errors get costlier for the decision-maker, or when the distribution gets

\footnotetext{
16 It is worthwhile to note that in the example presented in BM on the optimality of homogeneous panel, they assume quadratic loss and uniform distribution of states, a specification that satisfies the two conditions stated in Proposition 3.

17 To simplify the computation, this example features a discontinuous $f(\theta)$, contrary to what we assume elsewhere. However, a close enough continuous approximation to $f$ defined here would deliver an example with similar properties.

18 In fact, we can show that when the loss function is quadratic, i.e., $v(|y-\theta|)=(y-\theta)^{2}$, a condition that is more general than the logconcavity of $f$ is sufficient for the optimality of the homogeneous panel. (The condition is as follows: denote $S(y)=\int_{0}^{y} \int_{0}^{\theta} F(t) d t d \theta$ and $T(y)=\int_{y}^{1} \int_{\theta}^{1}(1-F(t)) d t d \theta$. If $S$ and $T$ are log-concave, then a homogeneous panel is optimal for every $\alpha$ in $(0,1)$. The proof of this result is very similar to that of Proposition 3.) Moreover, numerical calculations indicate that under quadratic loss the homogeneous panel remains optimal even under several log-convex distributions that are commonly used in the literature (e.g., Beta with appropriate parameters).
} 
more concentrated around some state. Unfortunately, a general comparative statics result of this nature appears elusive. The problem loses tractability as a change in the distribution or the loss function affects $\rho^{*}$ not only directly, but also indirectly through the default actions. However, we can illustrate these effects when we change the density function $f$ or the loss function $v$ in certain specific ways and in somewhat more restrictive environments. We provide an intuitive description of the two results below while the formal statements and proofs are presented in Appendix B (as Propositions B1 and B2 respectively).

Assume that $f$ is symmetric, implying that $y_{01}^{*}=0.5$. Now, suppose we perturb the loss function $v(z)$ in such a way that it remains unchanged for errors $z \leq 0.5$ but the loss increases for errors larger than 0.5 . With this change in the loss function, the payoff from the diverse panel remains unaffected but that from the homogeneous panel goes down. Therefore, as the loss from large errors gets larger, the diverse panel becomes more likely to be optimal (see Proposition B1).

Next, assume that $v$ is quadratic and $f$ is symmetric. Suppose we consider a perturbation of $f$ that makes it less concentrated "in the middle" in the following way: keeping $f$ unchanged to the left of $y_{11}^{*}$ and right of $y_{00}^{*}$, we decrease the conditional variance of $\theta$ in the range $\left[y_{11}^{*}, y_{00}^{*}\right]$. This change does not affect the default actions in either panel, but the reduction in concentration of states near $y_{01}^{*}=0.5$ reduces the payoff to the diverse panel more than that of the homogeneous panel. On the other hand, keeping $f$ unchanged in the range $\left[y_{11}^{*}, y_{00}^{*}\right]$ while making it less concentrated "at the extremes" (i.e., left of $y_{11}^{*}$ and right of $y_{00}^{*}$ ) raises $\rho^{*}$, making the diverse panel more likely to be optimal (see Proposition B2).

One may also ask if Proposition 3 could be extended to characterize the optimal panel for any given $\rho$. Notice that if the correlation is negative $(\rho<0)$, under the conditions given in Proposition 3 a homogeneous panel would be optimal for any $\alpha$. The following proposition sheds light on the case of positive correlation $(\rho>0)$.

Proposition 4. Suppose that $f$ is log-concave and symmetric, and $v$ is quadratic. Then $\rho^{*}$ (as defined in Proposition 2) is non-negative and single-peaked in $\alpha$ : there exists $\alpha^{*} \in(0,1)$ such that $\rho^{*}(\alpha)$ is strictly increasing on $\left(0, \alpha^{*}\right)$ and strictly decreasing on $\left(\alpha^{*}, 1\right)$. Moreover, $\rho^{*} \rightarrow 0$ as $\alpha \rightarrow 0$ or 1 . Consequently, for any $\rho>0$, either the diverse panel is always optimal, or there exist cutoffs $\underline{\alpha}$ and $\bar{\alpha}$ such that a homogeneous panel is optimal when $\alpha \in(\underline{\alpha}, \bar{\alpha})$ and the diverse panel is optimal otherwise.

Observe that under the conditions specified in Proposition 4, for any positive $\rho$, no matter how small, the diverse panel will be optimal when $\alpha$ is small enough or large enough. On the other hand, the homogeneous panel remains optimal for moderate values of $\alpha$. The intuition is simple: The advantage of the homogeneous panel over the diverse panel comes from the fact that there is a second expert who may observe the state when the first expert does not. Notice that when $\rho$ is positive, the marginal value of the second expert in a homogeneous panel in comparison to the diverse panel is the lowest when $\alpha$ is either large or small. For large $\alpha$, it is already very likely that the first expert has observed the state, so the second expert in the homogeneous panel is useful with a very small probability. For small $\alpha$, it is very unlikely that the first expert has observed the state, so in the absence of correlation, it does not make much of a difference whether to form a homogeneous or a diverse panel; however, the positive correlation between the experts drives the value of the homogeneous panel down relative to the diverse panel. Therefore, the homogeneous panel is most effective for moderate values of $\alpha$. Moreover, a decrease in correlation enlarges the set of $\alpha$ for which the homogeneous panel is optimal (i.e. the interval $(\underline{\alpha}, \bar{\alpha})$ decreases with $\rho)$. For large enough values of correlation, it is possible that the diverse panel becomes optimal for all values of $\alpha$.

\subsection{Experts with different qualities}

In our model, we assume that the two experts have the same quality $(\alpha)$. While this assumption simplifies the analysis, none of our results actually depend on it. In contrast, suppose that the experts differ in their quality levels: expert $i$ 's quality is $\alpha_{i}, i \in\{A, B\}$, and $\alpha_{A} \neq \alpha_{B}$. We can argue that for any $\left(\alpha_{A}, \alpha_{B}\right) \in(0,1)^{2}$, both Propositions 2 and 3 continue to hold. ${ }^{19}$

This observation has a couple of important implications. First, it indicates that even if the panel consists of more than two experts, under conditions in Proposition 3 the optimal panel configuration is one where all experts have the same agenda. Note that from the decision-maker's point of view, having $n$ experts of qualities $\alpha_{1}, \ldots, \alpha_{n}$ with the same agenda $x$ is equivalent to having a single expert with agenda $x$ and quality $1-\prod_{i=1}^{n}\left(1-\alpha_{i}\right)$. Now, since Proposition 3 holds for all $\alpha_{A}$ and $\alpha_{B}$, it tells us that any panel where $m_{0}$ experts have agenda 0 and $m_{1}$ experts have agenda 1 can be improved upon by having a homogeneous panel where all $m_{0}+m_{1}$ experts have the same agenda ( 0 or 1 , as the case might be).

Second, in some environments, it allows us to explore how the difference in the experts' quality may impact the optimal panel choice.

Proposition 5. Fix $\alpha_{A}$ and $\alpha_{B}$. Suppose that $f(\theta)$ is continuous and bounded away from 0 . When the experts' types are independent $(\rho=0)$, a homogeneous panel is optimal if either $\frac{1-\alpha_{A}}{1-\alpha_{B}}$ or $\frac{1-\alpha_{B}}{1-\alpha_{A}}$ is close enough to 0 .

19 The proof is available from the authors upon request. 
The proposition above indicates that under any continuous and strictly positive prior distribution, a homogeneous panel is optimal if the experts sufficiently differ in their level of informedness. To see the intuition behind this finding, consider the case where $\alpha_{A}=1$ and $\alpha_{B}<1$. Let us fix $x_{A}=0$ and compare the panels $(0,1)$ and $(0,0)$. With either of these panels, full revelation will occur in equilibrium, because the default action will equal 1 , and expert $A$ will reveal every realization of $\theta$ (with the possible exception of $\theta=1$ ) with probability 1 . Therefore, both panels will result in zero expected loss for the decision-maker.

Now suppose that $\alpha_{A}$ decreases by a small amount. Regardless of $x_{B}$, this has two effects on the decision-maker's payoff. First, with a decrease in $\alpha_{A}$, the revelation set for expert $A$ shrinks as the default action decreases. This effect decreases the decision-maker's payoff. Second, even if the state lies in expert $A$ 's revelation set, it is now less likely to be revealed as expert $A$ is now less likely to be informed. This effect also reduces the decision-maker's payoff. Now, as the loss is 0 when $\alpha_{A}=1$, the first effect is of second-order and is dominated by the second effect. While the second effect increases the loss, it is less pronounced under a homogeneous panel. This is due to the fact that under a homogeneous panel the revelation set of expert $A$ coincides with that of expert $B$ (i.e., the state lying in expert $A$ 's revelation set would be revealed as long as at least one of the two experts is informed). Therefore, a sufficiently small decrease in $\alpha_{A}$ will make the homogeneous panel dominate the diverse panel.

\subsection{Costly information and noisy signals}

It is interesting to consider the implications of costly information acquisition in our model. Once we allow for the fact that experts may have to spend costly effort in acquiring information, some of our results may change. Such considerations uncover an added benefit of the diverse panel vis-a-vis the homogeneous panel-while the experts in a homogeneous panel suffer from free-riding concerns, there is no free riding in a diverse panel as the experts report over a disjoint set of states. However, once we account for these concerns, we may have multiple equilibria even with extreme panels. While a complete analysis of this issue is beyond the scope of this article, our preliminary analysis indicates that not only multiple equilibria may arise in such a setting, but also we cannot rule out the possibility that a non-extreme panel may be optimal. ${ }^{20}$ Moreover, the findings appear to be very sensitive to the properties of the utility functions of the experts (e.g., risk aversion), which have played little role in the current article. Nevertheless, the key trade-off with panel choice that we highlight here continues to play an important role even in such a complex environment.

Another interesting question is whether the findings of the model are robust to assuming that each expert observes a noisy signal of the state, not the state itself. Unfortunately, we were unable to produce a tractable extension of the model to this case that would produce sensible results. ${ }^{21}$ However, we were able to analyze the equilibria of a simplified model, where the state is binary ( 0 or 1 ), and each expert observes the correct state with probability $p>0.5$, and the wrong state otherwise. The experts' signals are independent conditional on the state. In the noiseless version of this model (with $p=1$ ), Proposition 2 holds, and the conclusion of Proposition 3 holds as long as the decision-maker's loss function is log-concave (i.e., under weaker conditions than those of Proposition 3). Moreover, for any given panel configuration and for all values of $\alpha$ and $\rho$, the decision-maker's payoff is continuous in $p$ at $p=1$. This implies that for any given $(\alpha, \rho)$-pair, there exists a threshold level of noise such that the payoff comparison between the different panel configurations is the same as in the noiseless model, as long as the amount of noise is below the threshold. (In particular, for any $\alpha$, the conclusion of Proposition 3 holds as long as the decision-maker's loss function is log-concave and the noise is small enough.) In this sense, the model is robust to the introduction of a small amount of noise. We were not able to formally prove an analog of Proposition 2 for the model with noise. However, our numerical analysis for the case when the decision-maker's loss function is quadratic indicates that the conclusion of Proposition 2 continues to hold.

\section{Conclusion}

In a persuasion game involving self-interested experts, common intuition suggests that seeking information from a panel of experts with diverse set of agendas leads to better decision-making. The experts with competing interests may restrict each others' ability to manipulate information-as one expert's unfavorable information may be favorable to the other, the former's incentive to conceal information is muted by the latter's incentive to reveal the truth. In this article, we argue that the issue of optimal panel design is considerably more nuanced. In particular, we find that a diverse panel is optimal if and only if the correlation between the experts' types is above a threshold. Moreover, when the experts' types are independent, under a wide set of environments the decision-maker is better off by consulting a homogeneous panel-i.e., one where the experts have identical and extreme preferences (prefer the highest or lowest action possible).

One may argue that the correlation in the experts' degree of "informedness" may depend on whether the experts rely on a common information source or whether there are multiple information sources and each expert has exclusive access to

\footnotetext{
20 We extended our model by assuming binary effort, absolute loss function for experts, and quadratic loss function for decision-maker.

21 For example, in the formulation where the experts' payoff functions are linear and each expert observes the true state with a positive probability, and a draw from a continuous distribution on $[0,1]$ otherwise, the setting with a homogeneous panel may fail to possess a symmetric Bayesian-Nash equilibrium in cutoff strategies.
} 
a specific source. Therefore, our findings suggest that the optimality of diverse agendas should be evaluated in conjunction with the diversity in information sources. In the presence of a large diversity in the experts' information sources, diversity in the experts' preferences is generally sub-optimal; and when there is a lack of diversity in information sources, it is indeed optimal to have experts with diverse preferences.

\section{Appendix A}

This appendix contains the proofs omitted in the text.

Proof of Proposition 2. Step 1. Given a default action $y \in[0,1]$, let $L(y)$ and $R(y)$ denote the expected loss from states to the left and right of $y$, respectively:

$$
L(y)=\int_{0}^{y} v(|y-\theta|) d F(\theta) \text { and } R(y)=\int_{y}^{1} v(|y-\theta|) d F(\theta) .
$$

Also, let $V_{x_{A} x_{B}}(\rho)$ be the expected decision-maker's equilibrium loss when the panel is $\left(x_{A}, x_{B}\right)$. Then part (b) of Proposition 1 implies that

$$
\begin{aligned}
& V_{01}(\rho)=\min _{y \in[0,1]}(1-\alpha) L(y)+(1-\alpha) R(y), \\
& V_{00}(\rho)=\min _{y \in[0,1]}\left[(1-\alpha)^{2}+\rho\right] L(y)+R(y), \\
& V_{11}(\rho)=\min _{y \in[0,1]} L(y)+\left[(1-\alpha)^{2}+\rho\right] R(y) .
\end{aligned}
$$

Step 2. Note that as $v(\cdot) \geq 0, V_{00}(\rho)$ and $V_{11}(\rho)$ are increasing in $\rho$ by the Envelope Theorem, whereas $V_{01}(\rho)$ is independent of $\rho$. When $\rho=\rho_{1}$, then $(1-\alpha)^{2}+\rho=1-\alpha$. This implies that $V_{01}\left(\rho_{1}\right)<\min \left\{V_{00}\left(\rho_{1}\right), V_{11}\left(\rho_{1}\right)\right\}$.

Step 3. The last step is to show that $V_{01}\left(\rho_{0}\right)>\min \left\{V_{00}\left(\rho_{0}\right), V_{11}\left(\rho_{0}\right)\right\}$. (The existence of $\rho^{*}$ would then follow by continuity.) Indeed, if $\alpha \in[0.5,1)$, then $\rho_{0}=-(1-\alpha)^{2}$, so $V_{00}\left(\rho_{0}\right)=V_{11}\left(\rho_{0}\right)=0<V_{01}\left(\rho_{0}\right)$. If $\alpha \in[0,0.5)$, then $\rho_{0}=-\alpha^{2}$, and $(1-\alpha)^{2}+\rho_{0}=1-2 \alpha$. In this case,

$$
\begin{aligned}
& V_{01}\left(\rho_{0}\right)-V_{00}\left(\rho_{0}\right) \\
& =\left[(1-\alpha) L\left(y_{01}^{*}\right)+(1-\alpha) R\left(y_{01}^{*}\right)\right]-\left[(1-2 \alpha) L\left(y_{00}^{*}\right)+R\left(y_{00}^{*}\right)\right] \\
& >\left[(1-\alpha) L\left(y_{01}^{*}\right)+(1-\alpha) R\left(y_{01}^{*}\right)\right]-\left[(1-2 \alpha) L\left(y_{01}^{*}\right)+R\left(y_{01}^{*}\right)\right] \\
& =\alpha\left[L\left(y_{01}^{*}\right)-R\left(y_{01}^{*}\right)\right] .
\end{aligned}
$$

Thus, if $L\left(y_{01}^{*}\right) \geq R\left(y_{01}^{*}\right)$, then $V_{01}\left(\rho_{0}\right)>V_{00}\left(\rho_{0}\right)$. If on the other hand, $L\left(y_{01}^{*}\right)<R\left(y_{01}^{*}\right)$, then by the same method we can show that $V_{01}\left(\rho_{0}\right)>V_{11}\left(\rho_{0}\right)$. Therefore, $V_{01}\left(\rho_{0}\right)>\min \left\{V_{00}\left(\rho_{0}\right), V_{11}\left(\rho_{0}\right)\right\}$.

Proof of Proposition 3. We show that the stated conditions are sufficient for a homogeneous panel to be optimal for all $\alpha$ when $\rho=0$. Hence, by Proposition 2 it follows that under the same conditions $\rho^{*}>0$ for all $\alpha$. The proof is given in the following steps:

Step 1. Let $V_{x_{A} x_{B}}:=V_{x_{A} x_{B}}(0)$, where $V_{x_{A} x_{B}}(\rho)$ is given by equation (3). For $t>0$, let

$$
\begin{aligned}
g(t) & =\min _{y \in[0,1]} \frac{1}{t}(1-\alpha)^{2} \int_{0}^{y} v(|y-\theta|) d F+t \int_{y}^{1} v(|y-\theta|) d F \\
& =\min _{y \in[0,1]} \frac{1}{t}(1-\alpha)^{2} L(y)+t R(y) .
\end{aligned}
$$

Then $V_{11}=g\left((1-\alpha)^{2}\right), V_{01}=g(1-\alpha)$, and $V_{00}=g(1)$. So, given $\alpha$, a homogeneous panel is optimal if and only if

$$
\min \left\{g\left((1-\alpha)^{2}\right), g(1)\right\} \leq g(1-\alpha) \text {. }
$$

Condition (5) holds for every $\alpha \in(0,1)$ if $g(t)$ is quasi-concave on $(0,1]$. Therefore, it is enough to prove that for any $\alpha \in(0,1)$, either: (a) $g^{\prime}(t) \geq 0$ for all $t \in(0,1)$; or (b) $g^{\prime}(t) \leq 0$ for all $t \in(0,1)$; or (c) there exists $t^{*} \in(0,1)$ such that $g^{\prime}(t) \geq 0$ for $t<t^{*}$ and $g^{\prime}(t) \leq 0$ for $t>t^{*}$.

Step 2. For any $\alpha \in(0,1)$, let $y^{*}(t)=\arg \min _{y \in[0,1]} g(t)$ be defined by the first-order conditions

$$
\frac{1}{t}(1-\alpha)^{2} L^{\prime}\left(y^{*}(t)\right)+t R^{\prime}\left(y^{*}(t)\right)=0 \text {. }
$$

By the Envelope Theorem, 


$$
g^{\prime}(t)=-\frac{1}{t^{2}}(1-\alpha)^{2} L\left(y^{*}(t)\right)+R\left(y^{*}(t)\right) .
$$

Hence, combining (6) and (7) we have:

$$
g^{\prime}(t) \gtreqless 0 \Leftrightarrow \ell\left(y^{*}(t)\right) \gtreqless r\left(y^{*}(t)\right),
$$

where for $y \in(0,1]$,

$$
\ell(y):=\frac{\int_{0}^{y} v^{\prime}(y-\theta) d F}{\int_{0}^{y} v(y-\theta) d F} \text { and } r(y):=\frac{\int_{y}^{1} v^{\prime}(\theta-y) d F}{\int_{y}^{1} v(\theta-y) d F} .
$$

Step 3. Since $L^{\prime \prime}(y)>0$ and $R^{\prime \prime}(y)>0$ by the convexity of $v$, the implicit function theorem applies, and the function $y^{*}(t)$ defined by (6) is differentiable. It is strictly increasing, because

$$
\frac{d y^{*}(t)}{d t}=\frac{t^{-2}(1-\alpha)^{2} L^{\prime}\left(y^{*}(t)\right)-R^{\prime}\left(y^{*}(t)\right)}{t^{-1}(1-\alpha)^{2} L^{\prime \prime}\left(y^{*}(t)\right)+t R^{\prime \prime}\left(y^{*}(t)\right)}>0 .
$$

Equation (6) implies that $\lim _{t \rightarrow 0} y^{*}(t)=0, \lim _{t \rightarrow+\infty} y^{*}(t)=1$. Therefore, to prove the quasi-concavity of $g(t)$, it is enough to show that either (a) for all $y \in(0,1), \ell(y) \geq r(y)$, or (b) for all $y \in(0,1), \ell(y) \leq r(y)$, or (c) there exists $y^{*} \in(0,1)$ such that $\ell(y) \geq r(y)$ if $y<y^{*}$ and $\ell(y) \leq r(y)$ if $y>y^{*}$. But to prove this, it is sufficient to show that $\ell(y)$ is decreasing and $r(y)$ is increasing on $(0,1)$. Indeed, if this is true and $\lim _{y \rightarrow 0} \ell(y) \leq r(0)$, then case (b) holds; if $\lim _{y \rightarrow 0} \ell(y)>r(0)$ and $\ell(1) \geq \lim _{y \rightarrow 1} r(y)$, then case (a) holds; finally, if $\lim _{y \rightarrow 0} \ell(y)>r(0)$ and $\ell(1)<\lim _{y \rightarrow 1} r(y)$, then case (c) holds.

Step 4a. Consider $\ell(y)$ first:

$$
\begin{aligned}
\ell^{\prime}(y) & =\frac{d}{d y}\left[\frac{\int_{0}^{y} v^{\prime}(y-\theta) d F}{\int_{0}^{y} v(y-\theta) d F}\right] \\
& =\frac{1}{\left(\int_{0}^{y} v(y-\theta) d F\right)^{2}}\left[\int_{0}^{y} v^{\prime \prime}(y-\theta) d F \int_{0}^{y} v(y-\theta) d F-\left(\int_{0}^{y} v^{\prime}(y-\theta) d F\right)^{2}\right] \\
& =\frac{1}{\left(\int_{0}^{y} v(y-\theta) d F\right)^{2}}\left[\int_{0}^{y} v^{\prime \prime}(y-\theta) d F \int_{0}^{y} v^{\prime}(y-\theta) \frac{F(\theta)}{f(\theta)} d F-\int_{0}^{y} v^{\prime}(y-\theta) d F \int_{0}^{y} v^{\prime \prime}(y-\theta) \frac{F(\theta)}{f(\theta)} d F\right] .
\end{aligned}
$$

The numerator is

$$
\begin{aligned}
& \int_{0}^{y} v^{\prime \prime}(y-\theta) d F \int_{0}^{y} v^{\prime}(y-\theta) \frac{F(\theta)}{f(\theta)} d F-\int_{0}^{y} v^{\prime}(y-\theta) d F \int_{0}^{y} v^{\prime \prime}(y-\theta) \frac{F(\theta)}{f(\theta)} d F \\
& =\int_{0}^{y} v^{\prime \prime}(y-\theta) d F \int_{0}^{y} v^{\prime \prime}(y-\theta) \frac{v^{\prime}(y-\theta)}{v^{\prime \prime}(y-\theta)} \frac{F(\theta)}{f(\theta)} d F-\int_{0}^{y} v^{\prime \prime}(y-\theta) \frac{v^{\prime}(y-\theta)}{v^{\prime \prime}(y-\theta)} d F \int_{0}^{y} v^{\prime \prime}(y-\theta) \frac{F(\theta)}{f(\theta)} d F .
\end{aligned}
$$

Dividing the above by $\left(\int_{0}^{y} v^{\prime \prime}(y-\theta) d F\right)^{2}$, which is positive, results in

$$
\begin{aligned}
& \int_{0}^{y} \frac{v^{\prime}(y-\theta)}{v^{\prime \prime}(y-\theta)} \frac{F(\theta)}{f(\theta)} \frac{v^{\prime \prime}(y-\theta)}{\int_{0}^{y} v^{\prime \prime}\left(y-\theta^{\prime}\right) d F\left(\theta^{\prime}\right)} d F-\int_{0}^{y} \frac{v^{\prime}(y-\theta)}{v^{\prime \prime}(y-\theta)} \frac{v^{\prime \prime}(y-\theta)}{\int_{0}^{y} v^{\prime \prime}\left(y-\theta^{\prime}\right) d F\left(\theta^{\prime}\right)} d F \\
& \quad \times \int_{0}^{y} \frac{F(\theta)}{f(\theta)} \frac{v^{\prime \prime}(y-\theta)}{\int_{0}^{y} v^{\prime \prime}\left(y-\theta^{\prime}\right) d F\left(\theta^{\prime}\right)} d F .
\end{aligned}
$$

For $x \in[0,1]$, let

$$
H(x)=\int_{0}^{x} \frac{v^{\prime \prime}(|y-\theta|)}{\int_{0}^{1} v^{\prime \prime}\left(\left|y-\theta^{\prime}\right|\right) d F\left(\theta^{\prime}\right)} d F(\theta) .
$$

The fact that $v^{\prime \prime}>0$ everywhere except possibly at 0 implies that $H(x)$ is a cumulative distribution function of a probability distribution on $[0,1]$ with density 


$$
h(x)=\frac{v^{\prime \prime}(|y-x|) f(x)}{\int_{0}^{1} v^{\prime \prime}\left(\left|y-\theta^{\prime}\right|\right) d F\left(\theta^{\prime}\right)} .
$$

Now, expression (10) can be rewritten as

$$
\begin{aligned}
& \frac{1}{H(y)} \int_{0}^{y} \frac{v^{\prime}(y-\theta)}{v^{\prime \prime}(y-\theta)} \frac{F(\theta)}{f(\theta)} d H-\frac{1}{H(y)} \int_{0}^{y} \frac{v^{\prime}(y-\theta)}{v^{\prime \prime}(y-\theta)} d H \times \frac{1}{H(y)} \int_{0}^{y} \frac{F(\theta)}{f(\theta)} d H \\
& =\mathbb{E}_{H}\left[\frac{v^{\prime}(y-\theta)}{v^{\prime \prime}(y-\theta)} \frac{F(\theta)}{f(\theta)} \mid \theta \leq y\right]-\mathbb{E}_{H}\left[\frac{v^{\prime}(y-\theta)}{v^{\prime \prime}(y-\theta)} \mid \theta \leq y\right] \mathbb{E}_{H}\left[\frac{F(\theta)}{f(\theta)} \mid \theta \leq y\right] \leq 0,
\end{aligned}
$$

as $\frac{v^{\prime}(y-\theta)}{v^{\prime \prime}(y-\theta)}$ is positive and decreasing in $\theta$, and $\frac{F(\theta)}{f(\theta)}$ is increasing in $\theta$ (since $f$ is log-concave). Therefore $\ell^{\prime}(y) \leq 0$.

Step 4b. Consider $r(y)$ now:

$$
\begin{aligned}
r^{\prime}(y)= & \frac{d}{d y}\left[\frac{\int_{y}^{1} v^{\prime}(\theta-y) d F}{\int_{y}^{1} v(\theta-y) d F}\right] \\
= & \frac{1}{\left(\int_{y}^{1} v(\theta-y) d F\right)^{2}}\left[-\int_{y}^{1} v^{\prime \prime}(\theta-y) d F \int_{y}^{1} v(\theta-y) d F+\left(\int_{y}^{1} v^{\prime}(\theta-y) d F\right)\right] \\
= & \frac{1}{\left(\int_{y}^{1} v(\theta-y) d F\right)^{2}}\left[-\int_{y}^{1} v^{\prime \prime}(\theta-y) d F \int_{y}^{1} v^{\prime}(\theta-y) \frac{1-F(\theta)}{f(\theta)} d F\right. \\
& \left.+\int_{y}^{1} v^{\prime}(\theta-y) d F \int_{y}^{1} v^{\prime \prime}(\theta-y) \frac{1-F(\theta)}{f(\theta)} d F\right] .
\end{aligned}
$$

Similarly to the calculations above, $r^{\prime}(y)$ has the same sign as

$$
\begin{aligned}
& -\int_{y}^{1} \frac{v^{\prime}(\theta-y)}{v^{\prime \prime}(\theta-y)} \frac{1-F(\theta)}{f(\theta)} \frac{v^{\prime \prime}(\theta-y)}{\int_{y}^{1} v^{\prime \prime}\left(\theta^{\prime}-y\right) d F\left(\theta^{\prime}\right)} d F+\int_{y}^{1} \frac{v^{\prime}(\theta-y)}{v^{\prime \prime}(\theta-y)} \frac{v^{\prime \prime}(\theta-y)}{\int_{y}^{1} v^{\prime \prime}\left(\theta^{\prime}-y\right) d F\left(\theta^{\prime}\right)} d F \\
& \quad \times \int_{y}^{1} \frac{1-F(\theta)}{f(\theta)} \frac{v^{\prime \prime}(\theta-y)}{\int_{y}^{1} v^{\prime \prime}\left(\theta^{\prime}-y\right) d F\left(\theta^{\prime}\right)} d F \\
& =-\mathbb{E}_{H}\left[\frac{v^{\prime}(\theta-y)}{v^{\prime \prime}(\theta-y)} \frac{1-F(\theta)}{f(\theta)} \mid \theta \geq y\right]+\mathbb{E}_{H}\left[\frac{v^{\prime}(\theta-y)}{v^{\prime \prime}(\theta-y)} \mid \theta \geq y\right] \mathbb{E}_{H}\left[\frac{1-F(\theta)}{f(\theta)} \mid \theta \geq y\right] \geq 0,
\end{aligned}
$$

as $\frac{v^{\prime}(\theta-y)}{v^{\prime \prime}(\theta-y)}$ is positive and increasing in $\theta$, and $\frac{1-F(\theta)}{f(\theta)}$ is decreasing in $\theta$ (since $f$ is log-concave). Therefore $r^{\prime}(y) \geq 0$.

Proof of Proposition 4. Step 1. The fact that $\lim _{\alpha \rightarrow 0} \rho^{*}(\alpha)=\lim _{\alpha \rightarrow 1} \rho^{*}(\alpha)=0$ follows from the condition that $\rho^{*}(\alpha) \in$ $\left[-\min \left\{\alpha^{2},(1-\alpha)^{2}\right\}, \alpha(1-\alpha)\right]$. The fact that $\rho^{*} \geq 0$ follows from Proposition 3 .

Step 2. Let us now prove that $\rho^{*}(\alpha)$ is single-peaked. The fact that $f$ is symmetric implies that for any $\rho$ and $\alpha$, $V_{00}=V_{11}$ and $y_{01}^{*}=\frac{1}{2}$.

Let $\pi_{01}=(1-\alpha)^{-1} V_{01}=L\left(y_{01}^{*}\right)+R\left(y_{01}^{*}\right)$ (note that $\pi_{01}$ does not depend on $\alpha$ ), and let $y^{*}(\alpha)=y_{00}^{*}\left(\alpha, \rho^{*}(\alpha)\right.$ ). Also, for brevity, let us write $L:=L\left(y^{*}(\alpha)\right), L^{\prime}:=\frac{d}{d y} L\left(y^{*}(\alpha)\right)$, and $L^{\prime \prime}:=\frac{d^{2}}{d y^{2}} L\left(y^{*}(\alpha)\right)$, and similarly for $R$. We know that $\left(\rho^{*}(\alpha), y^{*}(\alpha)\right)$ jointly satisfy

$$
\left((1-\alpha)^{2}+\rho^{*}\right) L+R=(1-\alpha) \pi_{01} ;\left((1-\alpha)^{2}+\rho^{*}\right) L^{\prime}+R^{\prime}=0 .
$$

Now, differentiating this system of equations one obtains:

$$
\frac{d \rho^{*}}{d \alpha}=2(1-\alpha)-\frac{\pi_{01}}{L} ; \frac{d y^{*}}{d \alpha}=\frac{\pi_{01} L^{\prime}}{L \mathcal{D}} ;
$$

where $\mathcal{D}=\left((1-\alpha)^{2}+\rho^{*}\right) L^{\prime \prime}+R^{\prime \prime}$. We want to show that $\rho^{*}(\alpha)$ has a unique global maximum on $[0,1]$. 
Step 3. The fact that $\lim _{\alpha \rightarrow 0} \rho^{*}(\alpha)=\lim _{\alpha \rightarrow 1} \rho^{*}(\alpha)=0$ and $\rho^{*}(\alpha) \geq 0$ implies that either $\rho^{*}(\alpha)$ is identically equal to zero, or is non-monotonic. In either case, there exists an $\alpha^{*} \in(0,1)$ such that $\frac{d}{d \alpha} \rho^{*}\left(\alpha^{*}\right)=0$.

It remains to be proved that such an $\alpha^{*}$ is unique. (Because $\lim _{\alpha \rightarrow 0} \rho^{*}(\alpha)=\lim _{\alpha \rightarrow 1} \rho^{*}(\alpha)=0, \rho^{*}(\alpha) \geq 0$, and $\rho^{*}$ is continuously differentiable, this will imply that $\rho^{*}(\alpha)$ is strictly increasing on $\left(0, \alpha^{*}\right)$ and strictly decreasing on $\left(\alpha^{*}, 1\right)$.)

Step 4. Note that $\frac{d}{d \alpha} \rho^{*}\left(\alpha^{*}\right)=0$ iff $\left(1-\alpha^{*}\right) L\left(y^{*}\left(\alpha^{*}\right)\right)=\frac{1}{2} \pi_{01}$; i.e., if we denote $G(\alpha):=(1-\alpha) L\left(y^{*}(\alpha)\right)$, then $G\left(\alpha^{*}\right)=$ $G(0)$. To show that such an $\alpha^{*}$ is unique, it is enough to show that $G(\alpha)$ is strictly concave.

Now,

$$
G^{\prime}(\alpha)=-L+\pi_{01}(1-\alpha) \frac{\left(L^{\prime}\right)^{2}}{L \mathcal{D}},
$$

and

$$
\begin{aligned}
G^{\prime \prime}(\alpha)= & -\frac{\left(L^{\prime}\right)^{2} \pi_{01}}{L \mathcal{D}}+\frac{\pi_{01}}{L^{2} \mathcal{D}^{2}}\left[\left\{-\left(L^{\prime}\right)^{2}+2(1-\alpha)\left(L^{\prime}\right)^{2} L^{\prime \prime} \frac{\pi_{01}}{L \mathcal{D}}\right\} L \mathcal{D}\right. \\
& \left.-(1-\alpha)\left(L^{\prime}\right)^{2} \pi_{01}\left\{\frac{\left(L^{\prime}\right)^{2}}{L}+\frac{L^{\prime}}{\mathcal{D}} \frac{d \mathcal{D}}{d y}-L^{\prime \prime}\right\}\right] .
\end{aligned}
$$

Dividing $G^{\prime \prime}(\alpha)$ by $\frac{\left(L^{\prime}\right)^{2} \pi_{01}}{L \mathcal{D}}>0$ we see that $G^{\prime \prime}<0$ iff:

$$
\begin{aligned}
& -1+\frac{1}{L \mathcal{D}}\left[L \mathcal{D}\left\{-1+2(1-\alpha) L^{\prime \prime} \frac{\pi_{01}}{L \mathcal{D}}\right\}-(1-\alpha) \pi_{01}\left\{\frac{\left(L^{\prime}\right)^{2}}{L}+\frac{L^{\prime}}{\mathcal{D}} \frac{d \mathcal{D}}{d y}-L^{\prime \prime}\right\}\right] \\
& =-2+(1-\alpha) \frac{\pi_{01}}{L \mathcal{D}}\left[2 L^{\prime \prime}-\frac{\left(L^{\prime}\right)^{2}}{L}-\frac{L^{\prime}}{\mathcal{D}} \frac{d \mathcal{D}}{d y}+L^{\prime \prime}\right]<0 .
\end{aligned}
$$

Since $v$ is quadratic, $L^{\prime \prime \prime}=R^{\prime \prime \prime}=0$. So, $\frac{d \mathcal{D}}{d y}=\left((1-\alpha)^{2}+\rho^{*}\right) L^{\prime \prime \prime}+R^{\prime \prime \prime}=0$, and hence, it is enough to establish the following two inequalities:

(a) $(1-\alpha) \frac{\pi_{01} L^{\prime \prime}}{L \mathcal{D}}<1$; and (b) $L^{\prime \prime}<\frac{\left(L^{\prime}\right)^{2}}{L}$.

Step 5. Using the fact that $(1-\alpha) \pi_{01}=\left((1-\alpha)^{2}+\rho^{*}\right) L+R$, inequality (a) reduces to:

$$
\left[\left((1-\alpha)^{2}+\rho^{*}\right) L+R\right] L^{\prime \prime}<\left[\left((1-\alpha)^{2}+\rho^{*}\right) L^{\prime \prime}+R^{\prime \prime}\right] L \Leftrightarrow L^{\prime \prime} / L<R^{\prime \prime} / R
$$

This inequality follows from the fact that $R(1-y) \equiv L(y)$ (as $f$ is symmetric), $y^{*}(\alpha) \geq \frac{1}{2}$, and $\frac{L^{\prime \prime}}{L}=\frac{L^{\prime \prime}}{L^{\prime}} \times \frac{L^{\prime}}{L}$ is decreasing (the proof that $L^{\prime} / L$ is decreasing can be found in the proof of Proposition 3; the proof that $L^{\prime \prime} / L^{\prime}$ is decreasing is identical). Inequality (b) simply requires $L^{\prime \prime} / L^{\prime}<L^{\prime} / L$, which follows from the fact that $L^{\prime} / L$ decreasing.

Proof of Proposition 5. Step 1. First, let us extend the technique behind the proof of steps 1 and 2 of the proof of Proposition 3 to the case where $\alpha_{A} \neq \alpha_{B}$. This will give us a necessary and sufficient condition for a homogeneous panel to be optimal for a given pair $\left(\alpha_{A}, \alpha_{B}\right)$.

Let

$$
\tilde{g}(t)=\min _{y \in[0,1]} \frac{1}{t}\left(1-\alpha_{A}\right)\left(1-\alpha_{B}\right) L(y)+t R(y) .
$$

Then the decision-maker's loss with the panel $\left(x_{A}, x_{B}\right), \tilde{V}_{x_{A} x_{B}}$, is given by $\tilde{V}_{11}=\tilde{g}\left(\left(1-\alpha_{A}\right)\left(1-\alpha_{B}\right)\right), \tilde{V}_{01}=\tilde{g}\left(1-\alpha_{B}\right), \tilde{V}_{10}=$ $\tilde{g}\left(1-\alpha_{A}\right)$, and $\tilde{V}_{00}=\tilde{g}(1)$. Therefore, a homogeneous panel is optimal if and only if $\min \left\{\tilde{g}\left(\left(1-\alpha_{A}\right)\left(1-\alpha_{B}\right)\right), \tilde{g}(1)\right\} \leq$ $\min \left\{\tilde{g}\left(1-\alpha_{A}\right), \tilde{g}\left(1-\alpha_{B}\right)\right\}$.

Let $\tilde{y}^{*}(t)=\arg \min _{y \in[0,1]} \tilde{g}(t)$ be defined by the first-order conditions

$$
\frac{1}{t}\left(1-\alpha_{A}\right)\left(1-\alpha_{B}\right) L^{\prime}\left(\tilde{y}^{*}(t)\right)+t R^{\prime}\left(\tilde{y}^{*}(t)\right)=0 .
$$

Then the default action with the panel $\left(x_{A}, x_{B}\right), \tilde{y}_{x_{A} x_{B}}^{*}$, is given by $\tilde{y}_{11}^{*}=\tilde{y}^{*}\left(\left(1-\alpha_{A}\right)\left(1-\alpha_{B}\right)\right), \tilde{y}_{01}^{*}=\tilde{y}^{*}\left(1-\alpha_{B}\right), \tilde{y}_{10}^{*}=$ $\tilde{y}^{*}\left(1-\alpha_{A}\right)$, and $\tilde{y}_{00}^{*}=\tilde{y}^{*}(1)$. It is easy to extend the proof of Proposition 3 to show that $\tilde{g}^{\prime}(t) \gtreqless 0 \Leftrightarrow \ell\left(\tilde{y}^{*}(t)\right) \gtreqless r\left(\tilde{y}^{*}(t)\right)$, where $\ell(y)$ and $r(y)$ are defined by equation (9).

Step 2. Let us now examine the properties of the functions $\ell(y)$ and $r(y)$ when $y$ is close enough to 0 or 1 . This step will show that if $\tilde{y}_{11}^{*}$ and $\tilde{y}_{10}^{*}$ are small enough, and $\tilde{y}_{00}$ and $\tilde{y}_{01}$ are large enough, then $\tilde{V}_{11}<\tilde{V}_{10}$ and $\tilde{V}_{00}<\tilde{V}_{01}$, meaning that a homogeneous panel is optimal. 
Integrating by parts the numerator and the denominator of $\ell(y)$ and using the fact that $v^{\prime}(0)=0$ one obtains:

$$
\lim _{y \rightarrow 0} \frac{1}{\ell(y)}=\lim _{y \rightarrow 0} \frac{\int_{0}^{y} v^{\prime}(y-\theta) F(\theta) d \theta}{\int_{0}^{y} v^{\prime}(y-\theta) f(\theta) d \theta} \leq \lim _{y \rightarrow 0} \frac{\max _{x \in[0, y]} F(x) \int_{0}^{y} v^{\prime}(y-\theta) d \theta}{\min _{x \in[0, y]} f(x) \int_{0}^{y} v^{\prime}(y-\theta) d \theta}=0,
$$

since $f(y)$ is bounded away from zero. Since $\ell(y)>0$, this implies that $\lim _{y \rightarrow 0} 1 / \ell(y)=0$, and $\lim _{y \rightarrow 0} \ell(y)=+\infty$. However,

$$
r(0)=\frac{\int_{0}^{1} v^{\prime}(y-\theta) d F}{\int_{0}^{1} v(y-\theta) d F}<\infty .
$$

Therefore, there exists $\varepsilon_{1}>0$ such that if $\tilde{y}^{*}(t)<\varepsilon_{1}$, then $\ell\left(\tilde{y}^{*}(t)\right)>r\left(\tilde{y}^{*}(t)\right)$, and $\tilde{g}^{\prime}(t)>0$. Convexity of $v$ implies that $\tilde{y}^{*}(t)$ is strictly increasing. So if $\tilde{y}_{11}^{*}=\tilde{y}^{*}\left(\left(1-\alpha_{A}\right)\left(1-\alpha_{B}\right)\right)<\tilde{y}_{10}^{*}=\tilde{y}^{*}\left(1-\alpha_{A}\right)<\varepsilon_{1}$, then $\tilde{y}^{*}(t)<\varepsilon_{1}$ for $t \in\left[\left(1-\alpha_{A}\right)(1-\right.$ $\left.\left.\alpha_{B}\right), 1-\alpha_{A}\right]$ and therefore $\tilde{g}(t)$ is increasing on this interval. This means that $\tilde{V}_{11}=\tilde{g}\left(\left(1-\alpha_{A}\right)\left(1-\alpha_{B}\right)\right)<\tilde{g}\left(1-\alpha_{A}\right)=\tilde{V}_{10}$.

Similarly, $\lim _{y \rightarrow 1} r(y)=+\infty$ and $r(1)<\infty$. This implies that there exists $\varepsilon_{2}>0$ such that if $\tilde{y}^{*}(t)>1-\varepsilon_{2}$, then $\tilde{g}^{\prime}(t)<$ 0 . So if $\tilde{y}_{00}=\tilde{y}^{*}(1)>\tilde{y}_{01}=\tilde{y}^{*}\left(1-\alpha_{B}\right)>1-\varepsilon_{2}$, then $\tilde{y}^{*}(t)>1-\varepsilon_{2}$ for $t \in\left[1-\alpha_{B}, 1\right]$ and therefore $\tilde{g}(t)$ is decreasing on this interval. This means that $\tilde{V}_{00}=\tilde{g}(1)<\tilde{g}\left(1-\alpha_{B}\right)=\tilde{V}_{01}$.

Step 3. In this step, we will show that by driving $\max \left\{\left(1-\alpha_{A}\right)\left(1-\alpha_{B}\right), \frac{1-\alpha_{A}}{1-\alpha_{B}}\right\}$ to 0 , we can make $\tilde{y}_{11}^{*}$ and $\tilde{y}_{10}^{*}$ arbitrarily close to 0 , and $\tilde{y}_{00}$ and $\tilde{y}_{01}$ arbitrarily close to 1 .

Let $\varepsilon=\min \left\{\varepsilon_{1}, \varepsilon_{2}\right\}$. It remains to show that there exists $\delta>0$ such that if

$$
\max \left\{\left(1-\alpha_{A}\right)\left(1-\alpha_{B}\right), \frac{1-\alpha_{A}}{1-\alpha_{B}}\right\}<\delta,
$$

then $\tilde{y}_{11}^{*}<\tilde{y}_{10}^{*}<\varepsilon$ and $1-\varepsilon<\tilde{y}_{01}^{*}<\tilde{y}_{00}^{*}$. For $\beta>0$, let

$$
\begin{aligned}
& y_{0}(\beta)=\arg \min _{y \in[0,1]} \beta \int_{0}^{y} v(y-\theta) d F+\int_{y}^{1} v(\theta-y) d F, \\
& y_{1}(\beta)=\arg \min _{y \in[0,1]} \int_{0}^{y} v(y-\theta) d F+\beta \int_{y}^{1} v(\theta-y) d F .
\end{aligned}
$$

The first-order conditions for $y_{0}(\beta)$ and $y_{1}(\beta)$ are

$$
\begin{gathered}
\beta \int_{0}^{y_{0}(\beta)} v^{\prime}\left(y_{0}(\beta)-\theta\right) d F=\int_{y_{0}(\beta)}^{1} v^{\prime}\left(\theta-y_{0}(\beta)\right) d F, \\
\int_{0}^{y_{1}(\beta)} v^{\prime}\left(y_{1}(\beta)-\theta\right) d F=\beta \int_{y_{1}(\beta)}^{1} v^{\prime}\left(\theta-y_{1}(\beta)\right) d F .
\end{gathered}
$$

These conditions imply that $\lim _{\beta \rightarrow 0} y_{0}(\beta)=1, \lim _{\beta \rightarrow 0} y_{1}(\beta)=0$.

For any $\alpha_{A}, \alpha_{B} \in(0,1)$, note that $\tilde{y}_{00}^{*}=y_{0}\left(\left(1-\alpha_{A}\right)\left(1-\alpha_{B}\right)\right)$, $\tilde{y}_{01}^{*}=y_{0}\left(\frac{1-\alpha_{A}}{1-\alpha_{B}}\right), \quad \tilde{y}_{10}^{*}=y_{1}\left(\frac{1-\alpha_{A}}{1-\alpha_{B}}\right)$, and $\tilde{y}_{11}^{*}=$ $y_{1}\left(\left(1-\alpha_{A}\right)\left(1-\alpha_{B}\right)\right)$. It is straightforward to extend Proposition 1 to show that for any $\alpha_{A}, \alpha_{B} \in(0,1), \tilde{y}_{11}^{*}<\tilde{y}_{10}^{*}$ and $\tilde{y}_{01}^{*}<\tilde{y}_{00}^{*}$. Therefore, for any $\varepsilon>0$, there exists $\delta>0$ such that if $\max \left\{\left(1-\alpha_{A}\right)\left(1-\alpha_{B}\right), \frac{1-\alpha_{A}}{1-\alpha_{B}}\right\}<\delta$, then $\tilde{y}_{11}^{*}<\tilde{y}_{10}^{*}<\varepsilon$ and $1-\varepsilon<\tilde{y}_{01}^{*}<\tilde{y}_{00}^{*}$.

\section{Appendix B}

In this appendix, we present two propositions formalizing how changes in the loss function $v$ and density $f$ affect panel choice.

Proposition B1. Let $f$ be symmetric and consider a class of loss functions $v(x ; \gamma): R_{+} \times \Gamma \rightarrow R_{+}$(where $\Gamma$ is an open, convex subset of $R$ ) that are differentiable in $(x, \gamma)$, strictly increasing and convex in $x$, and such that $v(0, \gamma)=v^{\prime}(0, \gamma)=0$. Suppose that if $\gamma_{0}<\gamma_{1}$, then $v\left(x, \gamma_{0}\right)=v\left(x, \gamma_{1}\right)$ for $x \in[0,0.5]$ and $v\left(x, \gamma_{0}\right)<v\left(x, \gamma_{1}\right)$ for $x>0.5$. Then $\rho^{*}$ is decreasing in $\gamma$. That is, as the loss from errors that are larger than $1 / 2$ gets larger, the diverse panel becomes more likely to be optimal. 
Proof. Let $\rho^{*}(\gamma)$ be the value of $\rho^{*}$ (as defined by Proposition 2) that corresponds to $v(x ; \gamma)$, and let $y^{*}(\gamma)$ be the value of $y_{00}^{*}$ that corresponds to $v(x ; \gamma)$ and $\rho=\rho^{*}(\gamma)$. Then $\left(y^{*}(\gamma), \rho^{*}(\gamma)\right)$ satisfy:

$$
\begin{gathered}
\left((1-\alpha)^{2}+\rho^{*}\right) \int_{0}^{y^{*}} v\left(y^{*}-\theta, \gamma\right) d F+\int_{y^{*}}^{1} v\left(\theta-y^{*}, \gamma\right) d F=V_{01}, \\
\left((1-\alpha)^{2}+\rho^{*}\right) \int_{0}^{y^{*}} v^{\prime}\left(y^{*}-\theta, \gamma\right) d F-\int_{y^{*}}^{1} v^{\prime}\left(\theta-y^{*}, \gamma\right) d F=0,
\end{gathered}
$$

where $v^{\prime}$ is the partial derivative of $v$ with respect to the first variable.

Differentiating the first equation of (11) with respect to $\gamma$ and making use of the second equation, we obtain

$$
\begin{aligned}
& \frac{\partial}{\partial \gamma}\left[\left((1-\alpha)^{2}+\rho^{*}\right) \int_{0}^{y^{*}} v\left(y^{*}-\theta, \gamma\right) d F+\int_{y^{*}}^{1} v\left(\theta-y^{*}, \gamma\right) d F-V_{01}\right] \\
& \quad+\frac{\partial}{\partial \rho}\left[\left((1-\alpha)^{2}+\rho^{*}\right) \int_{0}^{y^{*}} v\left(y^{*}-\theta, \gamma\right) d F+\int_{y^{*}}^{1} v\left(\theta-y^{*}, \gamma\right) d F-V_{01}\right] \frac{d \rho^{*}}{d \gamma}=0 .
\end{aligned}
$$

Therefore,

$$
\operatorname{sign} \frac{d \rho^{*}}{d \gamma}=-\operatorname{sign} \frac{\partial}{\partial \gamma}\left[\left((1-\alpha)^{2}+\rho^{*}\right) \int_{0}^{y^{*}} v\left(y^{*}-\theta, \gamma\right) d F+\int_{y^{*}}^{1} v\left(\theta-y^{*}, \gamma\right) d F-V_{01}\right] .
$$

Using the second equation of (11) again, we obtain

$$
\begin{aligned}
& \frac{\partial}{\partial \gamma}\left[\left((1-\alpha)^{2}+\rho^{*}\right) \int_{0}^{y^{*}} v\left(y^{*}-\theta, \gamma\right) d F+\int_{y^{*}}^{1} v\left(\theta-y^{*}, \gamma\right) d F-V_{01}\right] \\
& =-\frac{\partial V_{01}}{\partial \gamma}+\frac{\partial}{\partial \gamma}\left[\int_{y^{*}}^{1} v\left(\theta-y^{*}, \gamma\right) d F\right]+\frac{\partial}{\partial \gamma}\left[\int_{0}^{y^{*}} v\left(y^{*}-\theta, \gamma\right) d F\right] \frac{\int_{y^{*}}^{1} v^{\prime}\left(\theta-y^{*}, \gamma\right) d F}{\int_{0}^{y^{*}} v^{\prime}\left(y^{*}-\theta, \gamma\right) d F}>0 .
\end{aligned}
$$

The inequality uses the facts that by the symmetry of $f, V_{01}=2 \int_{0}^{1 / 2} v(1 / 2-\theta, \gamma) d F$, and therefore $\frac{\partial V_{01}}{\partial \gamma}=0$; also (since $\left.y^{*} \geq 1 / 2\right), \frac{\partial}{\partial \gamma}\left[\int_{y^{*}}^{1} v\left(\theta-y^{*}, \gamma\right) d F\right]=0$; and $\frac{\partial}{\partial \gamma}\left[\int_{0}^{y^{*}} v\left(y^{*}-\theta, \gamma\right) d F\right]>0$. Therefore, $\frac{d \rho^{*}}{d \gamma}<0$.

Proposition B2. Suppose that $v$ is quadratic and consider a class of pdfs $f(\theta ; \varphi):[0,1] \times \Phi \rightarrow[0,1]$ (where $\Phi$ is an open, convex subset of $R$ ) that are symmetric and differentiable in $\theta$ (the parameter $\varphi$ measures the concentration of $f$, in the sense defined below). Fix $\rho \in\left(\rho_{0}, \rho_{1}\right)$ and consider two values of $\varphi, \varphi_{0}$ and $\varphi_{1}$. Let $y^{*}:=y_{00}^{*}\left(\rho, \varphi_{0}\right)$ be the default action with the left-homogeneous panel given $\rho$ and $\varphi_{0}$. Now, the following claims hold:

(i) Suppose that $f\left(\theta ; \varphi_{0}\right)=f\left(\theta ; \varphi_{1}\right)$ for $\theta \in\left[0,1-y^{*}\right) \cup\left(y^{*}, 1\right]$, and $V_{0}\left(\theta \mid\left[1-y^{*}, y^{*}\right]\right)<V_{1}\left(\theta \mid\left[1-y^{*}, y^{*}\right]\right)$ where $V_{0}$ and $V_{1}$ represent the variance of $\theta$ under $f\left(\theta ; \varphi_{0}\right)$ and $f\left(\theta ; \varphi_{1}\right)$ respectively (under these conditions $y^{*}:=y_{00}^{*}\left(\rho, \varphi_{0}\right)=y_{00}^{*}\left(\rho, \varphi_{1}\right)$ ). Then, if the homogeneous panel is optimal under $f\left(\theta ; \varphi_{0}\right)$, it is also optimal under $f\left(\theta ; \varphi_{1}\right)$. That is, keeping the distribution the same at the ends and making it less concentrated in the middle makes the homogeneous panel more likely to be optimal.

(ii) Suppose that $f\left(\theta ; \varphi_{0}\right)=f\left(\theta ; \varphi_{1}\right)$ for $\theta \in\left[1-y^{*}, y^{*}\right]$, and $V_{0}\left(\theta \mid\left[0,1-y^{*}\right]\right)<V_{1}\left(\theta \mid\left[0,1-y^{*}\right]\right)$ (therefore, $V_{0}\left(\theta \mid\left[y^{*}, 1\right]\right)<$ $V_{1}\left(\theta \mid\left[y^{*}, 1\right]\right)$ ). Suppose also that $E_{0}\left(\theta \mid\left[0,1-y^{*}\right]\right)=E_{1}\left(\theta \mid\left[0,1-y^{*}\right]\right)$, where $E_{0}$ and $E_{1}$ represent the mean of $\theta$ under $f\left(\theta ; \varphi_{0}\right)$ and $f\left(\theta ; \varphi_{1}\right)$ respectively (therefore, $E_{0}\left(\theta \mid\left[y^{*}, 1\right]\right)=E_{1}\left(\theta \mid\left[y^{*}, 1\right]\right) ;$ under these conditions $y^{*}:=y_{00}^{*}\left(\rho, \varphi_{0}\right)=$ $\left.y_{00}^{*}\left(\rho, \varphi_{1}\right)\right)$. Then, if the diverse panel is optimal under $f\left(\theta ; \varphi_{0}\right)$, it is also optimal under $f\left(\theta ; \varphi_{1}\right)$. That is, keeping the distribution unchanged in the middle and making it less concentrated at the ends makes the diverse panel more likely to be optimal.

Proof. For a given $\varphi \in\left\{\varphi_{0}, \varphi_{1}\right\}$, consider the difference in losses between the left-homogeneous and the diverse panels:

$$
\left(V_{00}-V_{01}\right)(\varphi)=\left((1-\alpha)^{2}+\rho\right) \int_{0}^{y^{*}}\left(y^{*}-\theta\right)^{2} d F(\theta ; \varphi)+\int_{y^{*}}^{1}\left(\theta-y^{*}\right)^{2} d F(\theta ; \varphi)-(1-\alpha) \int_{0}^{1}(0.5-\theta)^{2} d F(\theta ; \varphi)
$$




$$
\begin{aligned}
= & \left((1-\alpha)^{2}+\rho\right)\left[\int_{0}^{y^{*}} \theta^{2} d F(\theta ; \varphi)-2 y^{*} \int_{0}^{y^{*}} \theta d F(\theta ; \varphi)+\left(y^{*}\right)^{2} F\left(y^{*} ; \varphi\right)\right] \\
& +\int_{y^{*}}^{1} \theta^{2} d F(\theta ; \varphi)-2 y^{*} \int_{y^{*}}^{1} \theta d F(\theta ; \varphi)+\left(1-F\left(y^{*} ; \varphi\right)\right)\left(y^{*}\right)^{2}-(1-\alpha)\left[\int_{0}^{1} \theta^{2} d F(\theta ; \varphi)-\frac{1}{4}\right] .
\end{aligned}
$$

(Here $y^{*}=y_{00}^{*}\left(\rho, \varphi_{0}\right)=y_{00}^{*}\left(\rho, \varphi_{1}\right)$.) Let $\Delta=\left(V_{00}-V_{01}\right)\left(\varphi_{1}\right)-\left(V_{00}-V_{01}\right)\left(\varphi_{0}\right)$.

Proof of statement (i): Under the assumptions stated in part (i), $\int_{y^{*}}^{1} \theta^{2} d F\left(\theta ; \varphi_{0}\right)=\int_{y^{*}}^{1} \theta^{2} d F\left(\theta ; \varphi_{1}\right), \int_{y^{*}}^{1} \theta d F\left(\theta ; \varphi_{0}\right)=$ $\int_{y^{*}}^{1} \theta d F\left(\theta ; \varphi_{1}\right)$. Since by the symmetry of $f$, we have to have $\int_{0}^{1} \theta d F=0.5$, the latter implies that $\int_{0}^{y^{*}} \theta d F\left(\theta ; \varphi_{0}\right)=$ $\int_{0}^{y^{*}} \theta d F\left(\theta ; \varphi_{1}\right)$. Therefore,

$$
\begin{aligned}
\Delta & =\left((1-\alpha)^{2}+\rho\right)\left[\int_{0}^{y^{*}} \theta^{2} d F\left(\theta ; \varphi_{1}\right)-\int_{0}^{y^{*}} \theta^{2} d F\left(\theta ; \varphi_{0}\right)\right]-(1-\alpha)\left[\int_{0}^{1} \theta^{2} d F\left(\theta ; \varphi_{1}\right)-\int_{0}^{1} \theta^{2} d F\left(\theta ; \varphi_{0}\right)\right] \\
& =\left((1-\alpha)^{2}+\rho-(1-\alpha)\right)\left[\int_{0}^{y^{*}} \theta^{2} d F\left(\theta ; \varphi_{1}\right)-\int_{0}^{y^{*}} \theta^{2} d F\left(\theta ; \varphi_{0}\right)\right] \\
& <0 .
\end{aligned}
$$

So if $\left(V_{00}-V_{01}\right)\left(\varphi_{0}\right)<0$, then $\left(V_{00}-V_{01}\right)\left(\varphi_{1}\right)<0$.

Proof of statement (ii): Under the assumption stated in part (ii), $\int_{1-y^{*}}^{y^{*}} \theta d F\left(\theta ; \varphi_{0}\right)=\int_{1-y^{*}}^{y^{*}} \theta d F\left(\theta ; \varphi_{1}\right), \int_{1-y^{*}}^{y^{*}} \theta^{2} d F\left(\theta ; \varphi_{0}\right)=$ $\int_{1-y^{*}}^{y^{*}} \theta^{2} d F\left(\theta ; \varphi_{1}\right)$. We have assumed that

$$
\int_{0}^{1-y^{*}} \theta d F\left(\theta ; \varphi_{0}\right)=\int_{0}^{1-y^{*}} \theta d F\left(\theta ; \varphi_{1}\right) ;
$$

since $f$ is symmetric and we have to have $\int_{0}^{1} \theta d F=0.5$, this implies that $\int_{0}^{y^{*}} \theta d F\left(\theta ; \varphi_{0}\right)=\int_{0}^{y^{*}} \theta d F\left(\theta ; \varphi_{1}\right)$. Therefore,

$$
\begin{aligned}
\Delta= & \left((1-\alpha)^{2}+\rho\right)\left[\int_{0}^{1-y^{*}} \theta^{2} d F\left(\theta ; \varphi_{1}\right)-\int_{0}^{1-y^{*}} \theta^{2} d F\left(\theta ; \varphi_{0}\right)\right]+\int_{y^{*}}^{1} \theta^{2} d F\left(\theta ; \varphi_{1}\right)-\int_{y^{*}}^{1} \theta^{2} d F\left(\theta ; \varphi_{0}\right) \\
& -(1-\alpha)\left[\int_{0}^{1-y^{*}} \theta^{2} d F\left(\theta ; \varphi_{1}\right)+\int_{y^{*}}^{1} \theta^{2} d F\left(\theta ; \varphi_{1}\right)-\int_{0}^{1} \theta^{2} d F\left(\theta ; \varphi_{0}\right)-\int_{y^{*}} \theta^{2} d F\left(\theta ; \varphi_{0}\right)\right] \\
= & \left((1-\alpha)^{2}+\rho-(1-\alpha)\right)\left[\int_{0}^{1-y^{*}} \theta^{2} d F\left(\theta ; \varphi_{1}\right)-\int_{0}^{1-y^{*}} \theta^{2} d F\left(\theta ; \varphi_{0}\right)\right] \\
& +(1-(1-\alpha))\left[\int_{y^{*}}^{1} \theta^{2} d F\left(\theta ; \varphi_{1}\right)-\int_{y^{*}}^{1} \theta^{2} d F\left(\theta ; \varphi_{0}\right)\right]
\end{aligned}
$$

$>0$.

So if $\left(V_{00}-V_{01}\right)\left(\varphi_{0}\right)>0$, then $\left(V_{00}-V_{01}\right)\left(\varphi_{1}\right)>0$.

\section{References}

Banerjee, A., Somanathan, R., 2001. A simple model of voice. Quart. J. Econ. 116, 189-227.

Battaglini, M., 2002. Multiple referrals and multidimensional cheap talk. Econometrica 70, 1379-1401.

Beniers, K., Swank, O., 2004. On the composition of committees. J. Law, Econ., Organ. 20, 353-378.

Ben-Porath, E., Dekel, E., Lipman, B., 2017. Commitment and robustness in mechanisms with evidence. Mimeo, Boston University.

Bhattacharya, S., Mukherjee, A., 2013. Strategic information revelation when experts compete to influence. RAND J. Econ. 44, $522-544$. 
Cai, H., 2009. Costly participation and heterogeneous preferences in informational committees. RAND J. Econ. 40, $173-189$.

Chen, Y., Olszewski, W., 2014. Effective persuasion. Int. Econ. Rev. 55, 319-347.

Dewatripont, M., Tirole, J., 1999. Advocates. J. Polit. Economy 107, 1-39.

Dziuda, W., 2011. Strategic argumentation. J. Econ. Theory 146, 1362-1397.

Dye, R., 1985. Disclosure of nonproprietary information. J. Acc. Res. 23, 123-145.

Gentzkow, M., Kamenica, E., 2017. Competition in persuasion. Rev. Econ. Stud. 84 (1), 300-322.

Gerardi, D., Yariv, L., 2008. Costly expertise. Am. Econ. Rev. Pap. Proc. 98, 187-193.

Giovannoni, F., Seidmann, D., 2007. Secrecy, two-sided bias and the value of evidence. Games Econ. Behav. 59, $296-315$.

Glazer, J., Rubinstein, A., 2001. Debates and decisions: on a rationale of argumentation rules. Games Econ. Behav. 36, $158-173$.

Grossman, S., 1981. The informational role of warranties and private disclosure about product quality. RAND J. Econ. 16, 328-340.

Hagenbach, J., Koessler, F., Perez-Richet, E., 2014. Certifiable pre-play communication: full disclosure. Econometrica 82, $1093-1131$.

Hart, S., Kremer, I., Perry, M., 2017. Evidence games: truth and commitment. Amer. Econ. Rev. 107, $690-717$.

Jung, W., Kwon, Y., 1988. Disclosure when the market is unsure of information endowment of managers. J. Acc. Res. 26, $146-153$.

Kartik, N., Suen, W., Xu, F., 2017. Investment in concealable information by biased experts. RAND J. Econ. 48 (1), $24-43$.

Krishna, V., Morgan, J., 2001a. A model of expertise. Quart. J. Econ. 116, 747-775.

Krishna, V., Morgan, J., 2001b. Asymmetric information and legislative rules: some amendments. Amer. Polit. Sci. Rev. 95, 435-452.

Ladha, K., 1992. The Condorcet jury theorem, free speech, and correlated votes. Am. J. Polit. Sci. 36, 617-634.

Lipman, B., Seppi, D., 1995. Robust inference in communication games with partial provability. J. Econ. Theory 66, $370-405$.

Mathis, J., 2008. Full revelation of information in sender-receiver games of persuasion. J. Econ. Theory 143, 571-584.

Milgrom, P., 1981. Good news and bad news: representation theorems and applications. Bell J. Econ. 12, 380-391.

Milgrom, P., 2008. What the seller won't tell you: persuasion and disclosure in markets. J. Econ. Perspect. 22, $115-131$.

Milgrom, P., Roberts, J., 1986. Relying on the information of interested parties. RAND J. Econ. 17, 18-32.

Newton, J., 2014. Cheap talk and editorial control. B.E. J. Theor. Econ. 14, 1-25.

Okuno-Fujiwara, M., Postlewaite, A., Suzumura, K., 1990. Strategic information revelation. Rev. Econ. Stud. 57, $25-47$.

Perez-Richet, E., 2012. Competing with equivocal information. Mimeo, Sciences Po Paris.

Seidmann, D., Winter, E., 1997. Strategic information transmission with verifiable messages. Econometrica 65, $163-169$.

Shavell, S., 1994. Acquisition and disclosure of information prior to sale. RAND J. Econ. 25, 20-36.

Sher, I., 2011. Credibility and determinism in a game of persuasion. Games Econ. Behav. 71, 409-419.

Shin, H., 1994. The burden of proof in a game of persuasion. J. Econ. Theory 64, 253-264.

Shin, H., 1998. Adversarial and inquisitorial procedures in arbitration. RAND J. Econ. 29, 378-405.

Sward, E., 1989. Values, ideology, and the evolution of the adversary system. Indiana Law J. 64, 301-355.

Verrecchia, R., 1983. Discretionary disclosure. J. Acc. Econ. 5, 179-194. 